\title{
灯具が傾けて取り付けられた場合の 道路面等照度図の作成方法
}

\author{
正会員福 原稔*
}

\section{1. まえがき}

道路照明用灯具の上万に, 非対称配光をもつ灯具の配

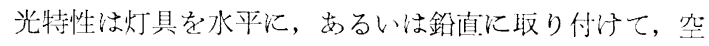
間の任意方向への光度を測定し，これを第1図のような 正弦等光度図住よって表方すのが常である.

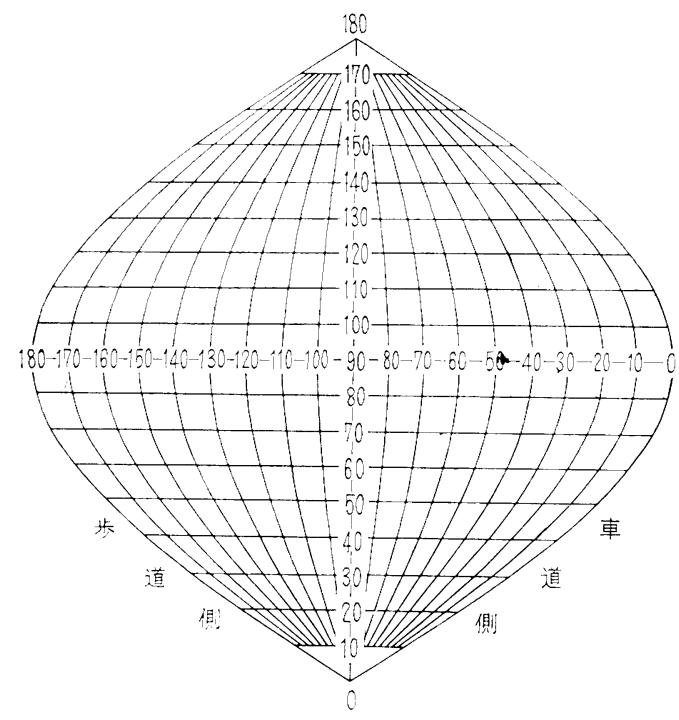

第 1 図正弦等光度図(等光度曲線省略)

ところでこの锺の灯具が実際汇使用されている状態を 調べてみると，水平（あるい性鉊直）に取り付忛られて いる場合より，支る估度傾けて取り付忊られている場合 の活らが多いが, この場合の配光特性は前の正弦等光度 図で表わされるものとは違って抢り, 前もって道路面等 照度図を描くことは不可能になる。

指定された取り付计角度に灯具を取り付计て配光を測 定し，正弦等光度図を描いてこれから道路面等照度图を 得るといら方法では, 指定の角度が変わるたび测定を やり直さなければならず，作業上の損失が大きい。

最近闸じ目的のために，従米の座標軸とは異なった灯 具の取り洲诌度の変化汇刘して, 一定の関係を保つよ

$\dagger$ Plotling of the Isolux Curve Chart for Road Construction Plan with Slanting Lighting Fixtures.

* 淞下電工明照明事業部

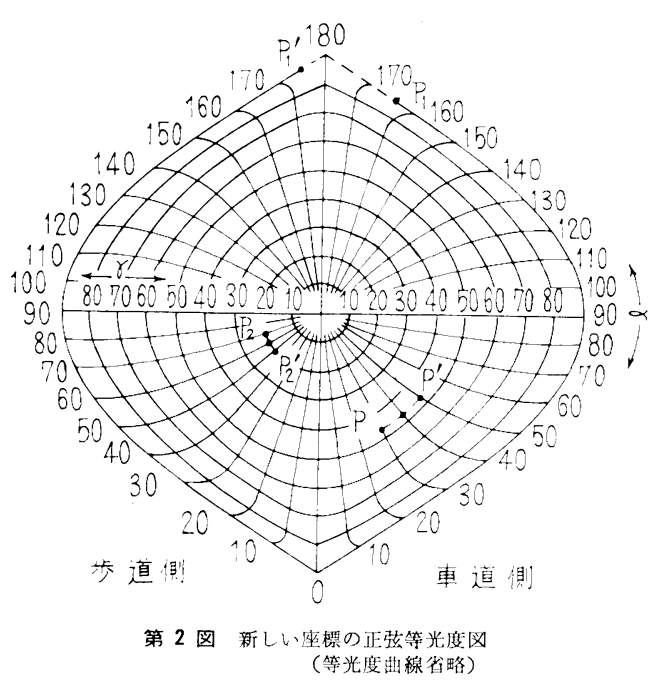

弓に考えた新しい座標軸をるつ正弦等光度図（第 2 図） を用いて簡便化しょうという方法が提案されている1). この方法では任意の取り付计角度汇そのまま用いること ができてその場合の正弦等光度図を描きうるが，座標の 変換を行ないながら，道路面等照度曲線を问時汇作成す ることはむずかしい。

上のような方法に刘して, 灯具の取り付悀度が变わ ると正弦等光度図上のある点の座標, 扎上びての点の道 路面上への投影点の座標がぞのような関係化従って変わ るかを計算で求めて扔く方法を紹介する.

\section{2. 図による説明と計算式}

第 3 図(a) は灯具の位置と道路の関係拉よびこれから 述べる計算に必要な点と, その座標を立体的汇表わした ものでX軸は道路の長手方向に, Y 軸は道路の幅方向に 相当する. $\mathrm{OO}^{\prime}$ は灯具の取り付高さで $\mathrm{O}$ 点に灯具 (光 中心)がある. 第 3 図(b) は同 (a) 図をX軸汇平行でか

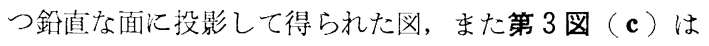
同 (a) 図を $\mathrm{Y}$ 軸化平行でから銷直な面化投影して得ら れた図である.点 $\mathrm{P}$ は点 $\mathrm{O}$ を心とした半径 $\mathrm{OO}^{\prime}$ の球面 上の任意の所沉あり，（a）図汇示すような極座標表示

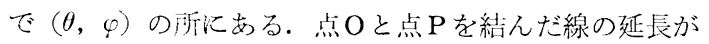
路面と交わる点が $\mathrm{Q}$ で, 路面上泎った直交座標表示で 


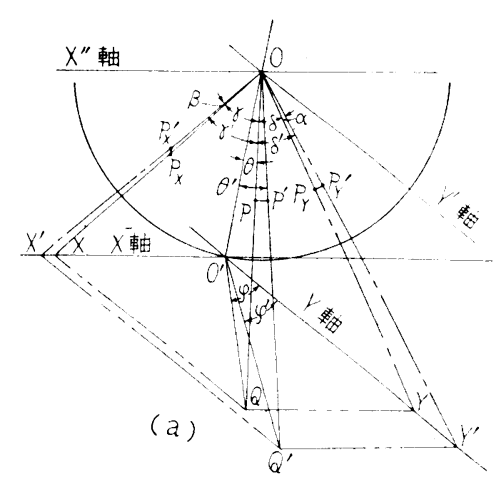

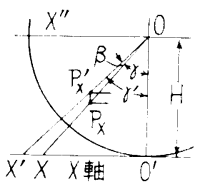

(b)

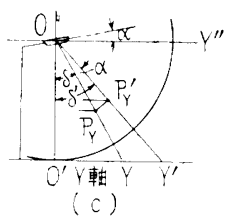

第 3 図道路と灯具の取り付以角度の関係を表わす図 (a)立体図 (b)道路の長手方向に平行で鉛淔な涌

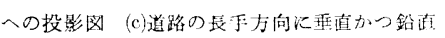
な面人の投影因

( $\mathrm{X}, \mathrm{Y}$ )の所他ある。(

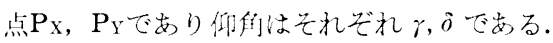

さて取り付外角度が $\alpha$ になるということは球 $\mathrm{O} か ゙$ 軸 $\mathrm{X}^{\prime \prime}$ のまわりを角 $\alpha$ だけ回転することに等しいから，これは (c)図にそのまま現われる。したがって仰角 $\partial^{\prime}=(\delta+\alpha)$ の 所に $\mathrm{P}_{\mathrm{Y}^{\prime}}$ が定まり， $\mathrm{P}$ 点の $\mathrm{X}$ 座標は変化しないことから

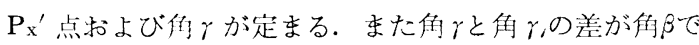
ある.このような関係汇従って点 $\mathrm{P}$ は点 $\mathrm{P}^{\prime}(\theta, \varphi)$ 亿点 $\mathrm{Q}$ は点 $\mathrm{Q}^{\prime}\left(\mathrm{X}^{\prime}, \mathrm{Y}^{\prime}\right)$ に移動する。結局点 $\mathrm{P}, \mathrm{Q}$ の座標の変化 を取り付惂度 $\alpha$ の関数として求めて括けば，水平汇取 り付忛た場合の正弦等光度図から任意角度に取り付けた 場合の道路面等照度図を直接描きうることがわかる。

棓算のつごう上取り付け储度が $\alpha$ の場合の路面上の任 意の点 $\mathrm{Q}^{\prime}\left(\mathrm{X}^{\prime}, \mathrm{Y}^{\prime}\right)$ と, 水平に取り付计た場合の正弦等 光度図上の点 $\mathrm{Q}^{\prime}$ 対応古る点 $\mathrm{P}(\theta, \varphi)$ の関係から求める ことにするが，式をより一般化するためX，Y扣よびX',

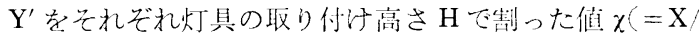
$\mathrm{H}), y(=\mathrm{Y} / \mathrm{H})$ 执よび $\chi^{\prime}\left(=\mathrm{X}^{\prime} / \mathrm{H}\right), y^{\prime}\left(=\mathrm{Y}^{\prime} / \mathrm{H}\right)$ を用 いる.

$$
\begin{aligned}
& y=\tan \delta \quad y^{\prime}=\tan \delta^{\prime}=\tan (\delta+\alpha) \\
& \therefore y^{\prime}=\frac{y^{\prime}-\tan \alpha}{1+y^{\prime} \tan \alpha} \\
& x=\tan \gamma \quad x^{\prime}=\tan \gamma^{\prime}=\tan (\gamma+\beta) \\
& \therefore \quad x=\frac{\sqrt{ } 1+\tan ^{2} \alpha}{1+y^{\prime} \tan \alpha} x^{\prime} \\
& \theta=\tan ^{-1} \sqrt{x^{2}+y^{2}}=\tan ^{-1} \\
& {\left[\frac{\sqrt{\left(1+\tan ^{2} \alpha\right) x^{\prime 2}+\left(y^{\prime}-\tan \alpha\right)^{2}}}{1+y^{\prime} \cdot \tan \alpha}\right]}
\end{aligned}
$$

$$
\varphi=\tan ^{-1}\left(\frac{x}{y}\right)=\tan ^{-1}\left[\frac{\sqrt{1+\tan ^{2} \alpha}}{y^{\prime}-\tan \alpha} \cdot x^{\prime}\right]
$$

(1)式は取り付け角度が $\alpha$ のときの路面上の任意の点 $\mathrm{Q}^{\prime}\left(\mathrm{X}^{\prime}, \mathrm{Y}^{\prime}\right)$ と, 水平に取り付けられたときの正弦等光度 図上の仰角 $\theta$ との, (2)式は方位角 $\varphi$ との関係式である.

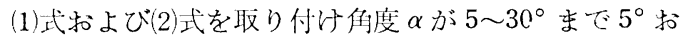
き計算したものが第 1 6 表である.

使用法を簡単に説明すると， $\alpha=5^{\circ}$ で取り付け高さ 10 $\mathrm{m}$ の場合に, $\mathrm{X}^{\prime}=10 \mathrm{~m}, \mathrm{Y}^{\prime}=6 \mathrm{~m}$ の点 $\left(x^{\prime}=1.0, y^{\prime}=\right.$ 0.6）の照度は第 1 表に従って水平に取り付外た場合の 正弦等光度図上の $\theta=47.2^{\circ}, \varphi=63.0^{\circ}$ の点の光度を読 又,これに距離の係数 $\left[=\left(10^{2}+6^{2}+10^{2}\right)^{-3 / 2}\right]$ を乗ずれ ば得られる.これを多くの点についてくり返せば氷平に 取り付けた場合の正弦等光度図から， $\alpha=5^{\circ}$ の場合の道 路面等照度図を描くことができる。このほか路面上の最 大・最小照度の存在する点を, 灯具配光と灯具配置上り 推定して照度を算出する遂点法に必用与ると，実测する ことなく取り付计角度を変克た場命の路面照度の均剂度 を容易に求めることができる。

\section{3. あとがき}

(1), (2), 式を適当な $\alpha$ について計算し表にしてお汁ば作 意の取り付忛角度の状態をつかむことができ，ての結果 灯具の最も有効な取り付战任度や適当なポール間隔を容 易に計算で求めうることは前節で述べたと扣りで女る.

夹测值とこの表を用いての計算值の比較を行な5場 合, 白熱灯や外い光灯では問題はないが, 水銀灯では次 のよ5な現象によって違いを生ずることがある。すなわ ら水銀灯の光出力は点灯方向によって影響され, 取り付 け角度が $0^{\circ}$ の場合上り $30^{\circ}$ の場合のほうが約 $5 \%$ 光出力 が增加すること拉よび水銀灯のアークの位㨁が点灯方向 によって変化する2)ので, ビームを作らせる形式の灯具 では配光特性が変わることを注意しなければならない。

なお $x^{\prime}, y^{\prime}$ の值は計算しやすい做を用いたが 0 汇近い 部分はも5少し細分した法らが良いが、これは は $y^{\prime}$ をパラメターとしたグラフを描いて補っている。

傾けて取り付忛場合の正弦等光度図を作成与るに は，参考文献(1)の方法が簡便であるが，式で対応させる と次のようになる（記号は第 3 図参照）

$\theta=\tan ^{-1}$

$$
\begin{aligned}
& {\left[\frac{\sqrt{\left(1+\tan ^{2} \alpha\right) \cdot \tan ^{2} \theta^{\prime} \cdot \sin ^{2} \varphi^{\prime}+\left(\tan \theta^{\prime} \cdot \cos \varphi^{\prime}-\tan \alpha\right)^{2}}}{1+\tan \theta^{\prime} \cdot \cos \varphi^{\prime} \cdot \tan \alpha}\right]} \\
& \varphi=\tan ^{-1}\left[\frac{\sqrt{ } 1+\tan ^{2} \alpha \cdot \tan \theta^{\prime} \cdot \sin \varphi^{\prime}}{\tan \theta^{\prime} \cdot \cos \varphi^{\prime}-\tan \alpha}\right]
\end{aligned}
$$

\section{参考文献}

(1) G.Mainwaring : Light and Lighting 55 (1962) 152

(2) 渡辺, 福原：昭36電気関係学会, 関西支部連大シン ポジウム I-4 


\begin{tabular}{|c|c|c|c|c|c|c|c|c|c|c|c|c|c|c|c|c|c|c|c|}
\hline \multirow{13}{*}{ 迹 } & : & $\begin{array}{l}-40 \\
-18 \%\end{array}$ & $\begin{array}{l}\infty 0 \\
\dot{\Phi} \\
\dot{\infty}\end{array}$ & $\begin{array}{l}\dot{\infty} \\
\dot{\infty}\end{array}$ & $\begin{array}{l}\infty \infty \\
\dot{\infty} \\
\dot{\infty}\end{array}$ & $\begin{array}{l}+\infty \\
\dot{\infty} \infty \\
\infty \infty\end{array}$ & $\begin{array}{c}m: 0 \\
\dot{\infty} \infty \dot{\infty}\end{array}$ & $\overrightarrow{\dot{\omega}}$ & 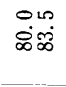 & $\begin{array}{l}\text { कें } \\
\text { शिं }\end{array}$ & $\begin{array}{l}\text { o. } \\
\text { Sig. }\end{array}$ & & $\begin{array}{l}6 \sigma 0 \\
\text { gise }\end{array}$ & $\begin{array}{l}n=0 \\
\text { sit }\end{array}$ & कूत & ㅁ. & 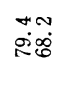 & $\begin{array}{l}\text { mo } \\
\text { giष }\end{array}$ & $\begin{array}{l}m \sim \\
\text { Sit }\end{array}$ \\
\hline & นึ. & 灾 & $\begin{array}{l}-10 \\
\dot{\infty} \dot{8}\end{array}$ & $\begin{array}{l}\infty \circ \\
\text { sim }\end{array}$ & సं.ं & 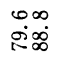 & $\begin{array}{l}+\infty \\
\text { si̊. }\end{array}$ & $\begin{array}{l}N \\
\text { gis } \\
\text { sid }\end{array}$ & 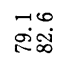 & $\begin{array}{l}0.0 \\
\text { s: } \\
\text { s. }\end{array}$ & 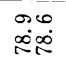 & $\begin{array}{ll}\infty \\
\infty \\
\infty\end{array}$ & 悉 & אid & $\begin{array}{l}\infty \\
\infty \\
\infty \\
\infty\end{array}$ & 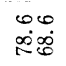 & D. & 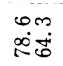 & $\begin{array}{l}0 \sim \\
\infty \sim \pi \\
\infty\end{array}$ \\
\hline & is & $\begin{array}{l}+\infty \\
\text { sis. }\end{array}$ & $\begin{array}{l}\text { Nus } \\
\text { sind }\end{array}$ & $\begin{array}{l}\infty \\
\infty m\end{array}$ & ro & $\begin{array}{l}n \pi n \\
\infty \infty \infty)\end{array}$ & $\ddot{\infty i \phi}$ & $\begin{array}{ll}N= \\
\infty\end{array}$ & $\rightarrow 0$ & 요. & $9 \div$ & $9 m$ & $\infty$. & & $\because$ & & & & \\
\hline & & & & & & $\approx \infty$ & $\approx \infty$ & 央 & $\approx \infty$ & 冓 & $\therefore N$ & $\approx$ 눙 & $\approx \Re$ & 듀 & $\therefore 8$ & $\therefore 8$ & Nㅕ & 청 & 충 \\
\hline & in & $\begin{array}{l}m \\
\infty i \infty \\
\infty \\
\infty\end{array}$ & $\begin{array}{l}\text { on } \\
\dot{\infty} \boldsymbol{i} \dot{y}\end{array}$ & $\stackrel{\infty}{\infty}$ & 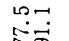 & \begin{tabular}{l}
$m \infty$ \\
\hdashline$\infty$ \\
$\therefore \infty$
\end{tabular} & \begin{tabular}{l}
-0 \\
\hdashline$i 0$
\end{tabular} & $\stackrel{0}{0.0}$ & $\begin{array}{l}\infty 0 \\
\dot{0}-1\end{array}$ & $\ddot{n}$ & or & $+\infty$ & & & & 6. & & & \\
\hline & s. & & & & तब & $\leqslant \infty \infty$ & $\therefore \infty$ & $\therefore \infty$ & $\stackrel{\circ}{\infty}$ & $2 \infty$ & 웅 & "20 & $\therefore$ & 180 & 80 & ¿ீं & i- & i⿱宀⿻心㇒丂 & isio \\
\hline & $\stackrel{0}{\circ}$ & $\begin{array}{l}\text { on } \\
\dot{\phi i s}\end{array}$ & $\begin{array}{l}100 \\
\dot{0} 0\end{array}$ & $\overrightarrow{0}=\vec{y}$ & $\begin{array}{l}O N \\
\dot{0}=1\end{array}$ & 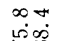 & 00 & 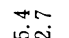 & mo. & NN & -6 & $-\infty$ & $\because$ & $-\infty$ & Nᄂ? & & & $\infty \pi$ & \\
\hline & 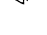 & & & 표 & & గீ冂ి & ஜூஜ & నంలు & LR & $\mathbb{L n}$ & $5 \pi$ & हூה & டி80 & 208 & غंठ் & शंठें & 료 & ம்is & 송ㅎㅁ \\
\hline & $\stackrel{18}{2}$ & تே & wis & $\dot{-1}$ & & $\begin{array}{l}\infty m \\
+i \infty\end{array}$ & $\stackrel{\infty}{\infty}$ & Lom & $+\infty$ & $\vec{m}$ & mo. & $\infty \infty$ & $\therefore$ & & . & & & & \\
\hline & $\dot{m}$ & $\therefore$ & 뉴. & ஜே & 125 & $\nabla+\infty$ & సోஷ & सేळ & $\therefore 8$ & 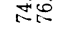 & संल & ¿i̊ & ind & ர்ष्ठ & זீஜ் & +ீठ் & संत् & ர்ष्ठ & เి่ \\
\hline & 요 & 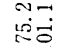 & $\stackrel{\infty}{\infty}$, & $\dot{x}$ & $i$ & $\infty \infty^{\circ}$ & rit & - & $+\infty$ & mis & & ? & & no. & & & & & \\
\hline & & $\because \varrho$ & 종 & $\approx \sigma$ & ジの & న & ఇాळ & ఇक् & జº & 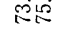 & నi் & ரீக் & న⿺辶 & लिषंठ & लंड் & mis & रें山ि & 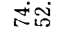 & ஓ்i \\
\hline & 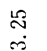 & تే & $\begin{array}{l}-\infty \\
\dot{\infty} \infty \\
\dot{\infty}\end{array}$ & $\begin{array}{l}m 0 \\
m i s\end{array}$ & $\begin{array}{l}90 \\
\text { i. }\end{array}$ & $\begin{array}{l}\text { No } \\
\text { îmo }\end{array}$ & 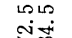 & $\vec{j}-\vec{i}$ & $m i$ & Nis & NN & 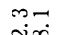 & $\forall N$ & om & $-\infty$ & O.n & mis & $0 \infty$. & O.N \\
\hline \multirow{2}{*}{ 相 } & & & & & & & & & & & $\therefore E$ & No & N & 젱요 & 죠 & నi⿱ & నః2 & isin & ని \\
\hline & $\stackrel{0}{\dot{m}}$ & 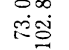 & $\begin{array}{l}10 \\
\text { Nig }\end{array}$ & 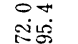 & Fr & mos & $\vec{F}$ & om & $\dot{a n}$ & $\begin{array}{l}\sigma N \\
\dot{m}\end{array}$ & or & $\exists$ & $m$ & nom & $\infty \varphi$ & $-\infty$ & $\ln \%$ & $\infty$ & \\
\hline \multirow[t]{2}{*}{ 溪 } & & & & & & & $\approx \infty$ & సळ & 25 & $\therefore i$ & 중 & $\therefore 8$ & $\approx 8$ & 28 & సడి & N芯 & $\mathbb{N} \overrightarrow{5}$ & NNo & ீீீ \\
\hline & & $\begin{array}{l}\sigma \infty \\
\vec{i} \\
\vec{i}\end{array}$ & $\begin{array}{l}\infty \infty \\
30 \\
i \infty\end{array}$ & $\begin{array}{ll}1-9 \\
0.8\end{array}$ & $\ddot{0}:-$ & $\therefore$ & sin & $\infty \pi$ & sin & $\begin{array}{l}\infty \\
\text { sidi }\end{array}$ & g. & $-\infty$ & $\forall n$ & 00 & or & m. & 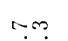 & & \\
\hline \multirow[b]{2}{*}{8} & & & & & & $\kappa_{\infty}^{\infty}$ & $0 \infty$ & 政 & 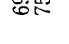 & हN & 88 & $8 \%$ & 86 & 요 & 요 요 & 츙 & EF & sid & సิ่ \\
\hline & $\stackrel{0}{i}$ & $\begin{array}{l}\infty \infty \\
\stackrel{0}{\circ} \dot{0} \\
i\end{array}$ & $\begin{array}{l}00 \\
80\end{array}$ & $\begin{array}{l}\operatorname{lng} \\
\sin \end{array}$ & 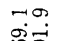 & Nis & $\begin{array}{ll}\operatorname{Lin} \\
\infty\end{array}$ & $+\infty$ & $\infty$ & 10r & $\sim \infty$ & $O N$ & mo. & $\leadsto$ & $\infty \infty$. & (अ) & $\neg \infty$ & $0 \pi$ & \\
\hline \multirow[t]{2}{*}{ 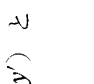 } & & & & 60 & & $6 \infty 0$ & $\sigma_{\infty}^{\infty}$ & o & 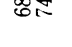 & do & 88 & మిరి & 용요 & 808 & 203 & io & 24 & $\therefore$ & i் \\
\hline & $\vec{d}$ & $\begin{array}{l}\text { ma } \\
\text { gitis }\end{array}$ & $\begin{array}{ll}6.4 \\
\infty\end{array}$ & $\begin{array}{l}0 \infty \\
\infty \\
\infty\end{array}$ & $=-1$ & $-m$ & क. & 90. & SL & $\because N$ & $\forall \sim$ & $\infty$ & N & $\infty$ & $\because ?$ & $\because$ & $m \sigma$ & & \\
\hline \multirow[t]{2}{*}{ z } & & & & & & రీ & $\varpi_{\infty}$ & $\Phi:$ & 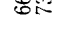 & 60 & 68 & 80 & 急 in & 象落 & $8 \overrightarrow{5}$ & 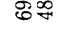 & হं⿺⿻一𠃋十 & $\dot{8}+\dot{7}$ & 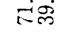 \\
\hline & $\underset{N}{N}$ & 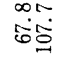 & $\ddot{\dot{\theta}}$ & 今े & 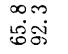 & $\begin{array}{l}m \infty \\
\ddot{\theta} \infty \dot{\infty}\end{array}$ & $\begin{array}{l}\overrightarrow{0} \cdot \overrightarrow{\dot{\theta}} \cdot \overrightarrow{0}\end{array}$ & $\begin{array}{l}n \% \\
1: 8 \\
180\end{array}$ & mi & nom & mo & मm & $\begin{array}{l}50 \\
0150\end{array}$ & 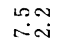 & $\vec{\infty}=$ & 90 & 菏 & $m$ & $\sigma=1$ \\
\hline \multirow[b]{2}{*}{ 形 } & & & & NN & & & & & & & & & & 6 in & & 67 & 67 & R\% & 요 \\
\hline & i & 80 & ச் & tં் & ชં & $\dot{6}$ & $\dot{\theta} \vec{\infty}$ & تृin & m.ं & 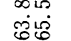 & 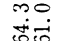 & $\begin{array}{l}\sigma \infty \\
+\infty\end{array}$ & 00 & mo. & $\dot{\sim}$ & 90 & $\infty-1$. & 00 & +6 \\
\hline \multirow{4}{*}{ in } & & & & & & & & & & & & 6 & 赑: & $\mathbb{8}$ & $6 \%$ & 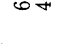 & ळm & 8ू. & 요 \\
\hline & -i & த் & ்ํ욤 & சंळ & छं் & 8 & छें & $\overrightarrow{8} \overline{8}$ & 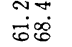 & 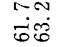 & 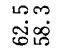 & $\begin{array}{l}\text { mo } \\
\text { :ंष्ठ }\end{array}$ & 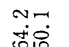 & $\begin{array}{l}-10 \\
\text { rof }\end{array}$ & $\dot{0 m}$ & $\begin{array}{l}0 \% \\
\therefore \circ \\
\therefore \circ\end{array}$ & $\begin{array}{l}0 \infty \\
\infty \\
\infty\end{array}$ & $\begin{array}{l}\sigma-1 \\
\infty \\
\infty\end{array}$ & $\infty$ \\
\hline & & & $\rightarrow 0$ & & & & & & & & & & & & & & & & \\
\hline & -7 & 6ळ & $8 \dot{8}$ & ஜ்த் & ம் & ப் & ஸिं & מ' & ம்ச் & म்ं & 8ำ & பं: & ن் & 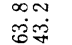 & $\begin{array}{l}\infty 00 \\
\dot{0} \dot{0} \dot{+}\end{array}$ & $\begin{array}{l}00 \\
\because \ddot{0}\end{array}$ & $\begin{array}{l}\sim 0 \\
\sim \infty\end{array}$ & No. & $\sim \infty$ \\
\hline \multirow{11}{*}{$\frac{\text { 彞 }}{\text { 船 }}$} & & $\infty-1$ & $0-1$ & & & & & & & & & & & & & & & & \\
\hline & 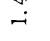 & ஸ் & ن்.8 & เீंத & ம்க & 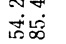 & 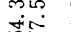 & $\begin{array}{l}\infty \circ \\
\text { tio }\end{array}$ & 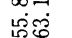 & 送 & $\begin{array}{l}m 60 \\
\infty \\
\infty\end{array}$ & sis & $\begin{array}{l}-\infty \\
-i n\end{array}$ & $\vec{m}$ & $m$ & $\because \Rightarrow$ & $\forall N$ & $+\infty$ & \\
\hline & & & & & & & & & & & & ம் & 68 & $6 \mathrm{~m}$ & 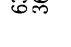 & $0 \%$ & $\Psi_{\infty}$ & 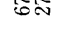 & రిశ్ \\
\hline & & 밍 & $\begin{array}{l}00 \\
\dot{m i n}\end{array}$ & $-\dot{m}$ & & வتं & Nor? & No. & & 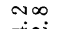 & & m.? & 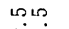 & -0. & טצ & N- & $1-18$ & om & \\
\hline & & 15 & in & & దूळ & 我交 & 앰요 & เू็ & พิ่ & 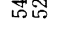 & แூ & 독 & 㥈冓 & бద్ల & ธิ่ี & 8ేं & ச்ष & $\dot{0} \dot{\Delta}$ & ஜ் \\
\hline & & $\vec{j} \dot{\vec{j}}$ & No. & $\begin{array}{l}\infty 0 \\
\infty 0\end{array}$ & $\because \because 0$ & ஜீ. & $\forall$ & ৩. & เ? & $m$ & $0-1$ & $\infty$ & 908 & $\because$ & L? & mos. & on & 100 & \\
\hline & & is $\approx$ & $=$ & F' & \% & অ゙⿱亠乂 & $\operatorname{lin}$ & 58 & Fin & Бே7 & ถู่ & ถீกิ & దీ & గిది & $\overline{6}$ & Bே. & ถู่สี่ & ถి & 69 \\
\hline & 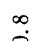 & $\begin{aligned}-0 \\
\infty \\
\infty\end{aligned}$ & $\stackrel{N N}{\sim \sim}$ & $\begin{array}{l}\text { or } \\
\text {-ios }\end{array}$ & कid & $\begin{array}{l}60 \\
\infty \\
\infty\end{array}$ & $\infty \infty$ & -1 [ & $\because+$ & & & $\sigma_{*}$ & & & & & & & \\
\hline & 0 & F & $+\widetilde{A}$ & $F$ & m\& & ల్ల & లిలి & 공 & 过奋 & $\mathscr{f}=$ & ஸ்கூ & ஜिं & 悉心 & ஸి & ธ்่ & ชิ่ & ț & 8 & 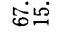 \\
\hline & & $\begin{array}{l}N \infty \\
\tilde{m} \infty \infty \\
\tilde{m} \infty\end{array}$ & ro & $\begin{array}{l}\sim \infty \\
\text { Yn }\end{array}$ & m. & 0. & $m \infty 0$ & $\because \therefore$ & $\because N$ & $N+$ & $\theta+$ & Nת & & & & & & & \\
\hline & & Fim & m. & 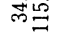 & लं & लंgi & พื่ & ம்ं & $\dot{\forall \dot{H}}$ & 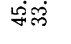 & 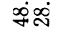 & ஸ่ં்ં & மீंत் & ஸ் & $\dot{\vec{x}}=$ & ت்ં & ச்ச் & ப் & $\dot{0}=$ \\
\hline \multirow{3}{*}{$\begin{array}{l}\text { io } \\
\text { II } \\
\sigma\end{array}$} & & or & NLO & ৩o & m. & m. & $6=$ & $\infty-1$ & $\forall *$ & దా. & s.os & -o & & & & & & & \\
\hline & & 역 & ri & సష్తి & స్ర్ల & ヘ̃ホ & ู่ં่ં & लिळ & దిని & รี่ి & S் & ค들 & ஜ்ர் & ம் & मिं & $\dot{\theta} 0$ & ஜூర & : & $80^{\circ}$ \\
\hline & $\approx$ & 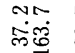 & $\begin{array}{c}-0 \\
\text { sic } \\
\text { win }\end{array}$ & $\begin{array}{l}\dot{9} \\
\dot{g} \dot{q}\end{array}$ & $\begin{array}{l}\text { mo } \\
\text { jig. }\end{array}$ & $\begin{array}{l}\text { or } \\
\text { ن. }\end{array}$ & $\begin{array}{l}\infty 6 \\
\text { Sid }\end{array}$ & : & Fin & $\dot{i} \vec{i}$ & $\begin{array}{l}\text { on } \\
\text { ion }\end{array}$ & $\begin{array}{l}\infty \pi \\
\infty \infty \infty\end{array}$ & กเด & 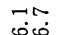 & 00 & Nm & $m$ & $+\infty$ & $\infty$ \\
\hline \multirow{2}{*}{$\vec{z}$} & & & & & & & & & & & & & & & & 6 & 0 & & \\
\hline & $\stackrel{0}{\circ}$ & 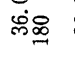 & is。 & : & ن் & 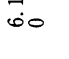 & $\stackrel{\infty}{\infty} \stackrel{\infty}{0}^{\circ}$ & i্ঠ) & ભ్లాం & 过o & 歺० & \$ా & : & 呫 & 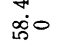 & :००० & :ై & : & : \\
\hline & & $\begin{array}{l}\ddot{0} \\
\dot{0} \\
1\end{array}$ & $\begin{array}{l}\ddot{0} \\
1 \\
1\end{array}$ & \begin{tabular}{l}
\multirow{2}{*}{} \\
$\dot{0}$ \\
1
\end{tabular} & 0 & 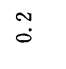 & $\stackrel{+}{0}$ & $\stackrel{0}{0}$ & $\stackrel{\infty}{\circ}$ & $\stackrel{\circ}{-i}$ & $\stackrel{\sim}{-}$ & $\stackrel{+}{-}$ & $\stackrel{0}{-}$ & $\stackrel{\infty}{-}$ & $\stackrel{0}{i}$ & $\begin{array}{l}\stackrel{2}{a} \\
\stackrel{\sim}{j}\end{array}$ & $\begin{array}{l}\stackrel{B}{6} \\
\text { i }\end{array}$ & $\begin{array}{l}\stackrel{R}{N} \\
\stackrel{N}{N}\end{array}$ & $\stackrel{\dot{m}}{ }$ \\
\hline
\end{tabular}




\begin{tabular}{|c|c|c|c|c|c|c|c|c|c|c|c|c|c|c|c|c|c|c|c|}
\hline 然 & $\dot{b}$ & 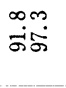 & ت্் & $\begin{array}{l}0.0 \\
\dot{\infty} \%\end{array}$ & $\begin{array}{l}\text { ¿̊. } \\
\dot{\infty} \dot{\sigma}\end{array}$ & $\begin{array}{l}+\infty \\
\dot{\infty} \infty \\
\dot{\infty} \infty \\
\infty\end{array}$ & $\begin{array}{l}0 . \\
\dot{\infty} \therefore \dot{\infty} \\
\dot{\infty} \infty\end{array}$ & $\begin{array}{l}00 \\
\text { Siष. }\end{array}$ & 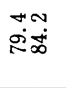 & $\begin{array}{l}N \infty \\
\text { Nis. }\end{array}$ & 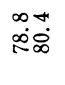 & $\infty$ & $\begin{array}{l}\text { mos } \\
\text { oico }\end{array}$ & $\begin{array}{l}N=-1 \\
\text { sing }\end{array}$ & $\begin{array}{l}0 m \\
\infty \infty 20\end{array}$ & 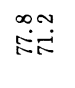 & 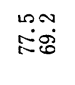 & $\begin{array}{l}\text { D-1. } \\
\text { i. }\end{array}$ & \begin{tabular}{l} 
No \\
\multirow{2}{*}{80}
\end{tabular} \\
\hline \multirow[t]{7}{*}{ 迸 } & is & $\begin{array}{l}0.5 \\
\text { i. }\end{array}$ & $\begin{array}{l}\infty \infty \\
\dot{\infty} 18 \\
\dot{\infty}\end{array}$ & $\begin{array}{l}\text { No } \\
\dot{\infty}\end{array}$ & $\begin{array}{l}\infty \infty \\
\text { शंजें }\end{array}$ & 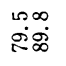 & 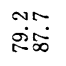 & 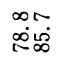 & $\begin{array}{l}100 \\
\infty \infty \infty \\
\infty \\
\infty \\
\infty\end{array}$ & 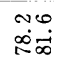 & $\begin{array}{l}500 \\
\text { Nis }\end{array}$ & Nㅗㅇ & : & 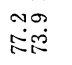 & 品 & 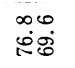 & ஸ் & 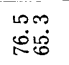 & $\begin{array}{l}m \infty \\
\dot{0}\end{array}$ \\
\hline & is & $\overrightarrow{\dot{\infty} \infty}$ & șïs & 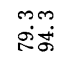 & 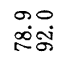 & $\begin{array}{l}\operatorname{Lr} \\
\infty \\
\infty \\
\infty\end{array}$ & 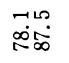 & 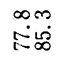 & 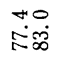 & 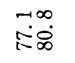 & $\begin{array}{l}\infty \pi \\
\dot{8} \infty \infty \\
i \infty\end{array}$ & $\begin{array}{l}\dot{0} \\
\dot{8} 0\end{array}$ & 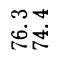 & 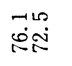 & $\begin{array}{l}\text { om } \\
\dot{\phi}\end{array}$ & ڤి- & $\begin{array}{l}\text { மீ- } \\
\text { ம்: }\end{array}$ & 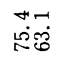 & $\begin{array}{l}\text { No } \\
\text { घं: }\end{array}$ \\
\hline & $\stackrel{n}{+}$ & "ే & 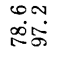 & $\begin{array}{l}\vec{\infty} \\
\dot{\infty}\end{array}$ & 총 & 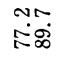 & 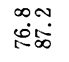 & 索政 & 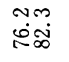 & $\begin{array}{l}\infty \infty \\
\text { pis }\end{array}$ & 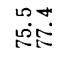 & $\begin{array}{l}\text { No } \\
\text { g்t. }\end{array}$ & $\begin{array}{l}\text { 으 } \\
\text { மำ }\end{array}$ & 悉- & $\begin{array}{l}0 N \\
\dot{1} \otimes\end{array}$ & 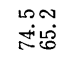 & $\begin{array}{l}\text { mo } \\
\text { ți் }\end{array}$ & $\begin{array}{l}\text { mo } \\
\text { संष्ठ }\end{array}$ & 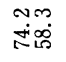 \\
\hline & $\stackrel{\circ}{+}$ & 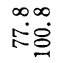 & $\begin{array}{l}\text { N.7. } \\
\text { 싱 }\end{array}$ & $\begin{array}{l}\text { జm } \\
\dot{\dot{s}}\end{array}$ & 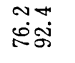 & जि: & $\begin{array}{l}\text { No } \\
\text { vis }\end{array}$ & $\begin{array}{l}\infty-\vec{j} \\
\dot{i}+\infty\end{array}$ & 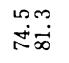 & $\begin{array}{l}-\infty \\
+\infty \\
+\infty \infty \\
+\infty\end{array}$ & $\begin{array}{l}\text { कo } \\
\text { mis }\end{array}$ & $\stackrel{m}{m}$ & تָi & 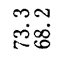 & $\begin{array}{l}-\infty \\
\dot{x}: \dot{\delta}\end{array}$ & 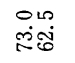 & $\begin{array}{l}\text { an } \\
\text { i் }\end{array}$ & $\begin{array}{l}\text { so } \\
\text { si.0. }\end{array}$ & $\begin{array}{l}\infty m \\
\text { sis. } \\
\text { sis }\end{array}$ \\
\hline & $\begin{array}{c}n \\
\substack{n \\
\infty}\end{array}$ & 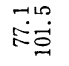 & $\begin{array}{l}+\infty \\
\dot{\theta} \otimes \infty \\
\dot{x} \infty\end{array}$ & 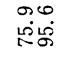 & $\begin{array}{l}m 0 \\
\text { ம் }\end{array}$ & $\begin{array}{l}\infty 0 \\
\dot{i} \infty \\
\dot{i} \infty\end{array}$ & 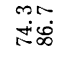 & $\tilde{\infty}_{\infty}^{\infty}$ & लिं & 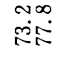 & $\begin{array}{l}\text { So } \\
\text { î̀ }\end{array}$ & $\tilde{N}$ & $\begin{array}{l}\text { मू. } \\
\text { 총ㅇ }\end{array}$ & $\begin{array}{l}\text { में } \\
\text { î: }\end{array}$ & $\begin{array}{l}\infty \infty \\
\text { జిర్ }\end{array}$ & 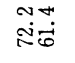 & 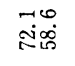 & 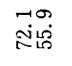 & 蔺 \\
\hline & 品 & $\begin{array}{l}\text { NM } \\
\text { ed }\end{array}$ & $\begin{array}{l}100 \\
\text { ம요 }\end{array}$ & $\begin{array}{l}\text { केनें } \\
+\dot{8}\end{array}$ & 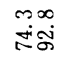 & 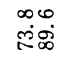 & 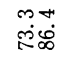 & $\begin{array}{l}\infty N \\
\dot{N} \infty \\
\dot{N} \infty\end{array}$ & 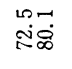 & 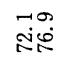 & $\begin{array}{l}\text { gr } \\
\dot{*}\end{array}$ & $\stackrel{\circ}{\stackrel{\leftrightarrow}{+}}$ & 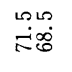 & 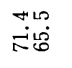 & $\begin{array}{l}m \infty \\
\dot{i} \\
\end{array}$ & 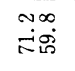 & $\begin{array}{l}m \infty \\
\dot{1} i \infty\end{array}$ & 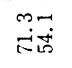 & $\begin{array}{l}+0 \\
\dot{\pi}\end{array}$ \\
\hline & $\underset{\infty}{\substack{\infty \\
\infty}}$ & $\begin{array}{l}\text { NN } \\
\text { î. }\end{array}$ & $\begin{array}{l}120 \\
+8 \\
+18\end{array}$ & $\begin{array}{l}\infty \infty \\
\text { लिष } \\
\text { लिष }\end{array}$ & $\begin{array}{l}\text { No } \\
\text { జి.్ర }\end{array}$ & 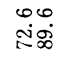 & $\overrightarrow{i \vec{D}}$ & 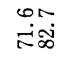 & 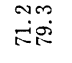 & $\begin{array}{l}90 \\
\dot{1} \\
\dot{8}\end{array}$ & $\hat{i} \tilde{i}$ & :0. & 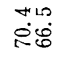 & $\begin{array}{l}m \infty \\
\dot{8}\end{array}$ & ن. & 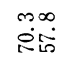 & $\begin{array}{l}\text { mos } \\
\text { i. }\end{array}$ & 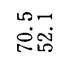 & \begin{tabular}{l}
010 \\
098 \\
\hdashline$i g$
\end{tabular} \\
\hline 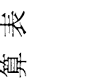 & $\stackrel{\circ}{\dot{m}}$ & تُ & ஜ்- & $\begin{array}{l}0-1 \\
\text { ล̇ं. }\end{array}$ & $\begin{array}{l}\text { gm } \\
\text { i் }\end{array}$ & \begin{tabular}{l} 
No \\
\multirow{i}{*}{$\infty$}
\end{tabular} & 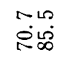 & $\begin{array}{l}\text { Nar } \\
\text { 尺्र }\end{array}$ & 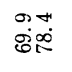 & $\begin{array}{l}00 \\
\dot{0}\end{array}$ & ه̇ & $\begin{array}{l}N N \\
\text { side }\end{array}$ & 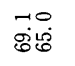 & تే: & 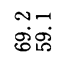 & $\begin{array}{l}m-2 \\
\text { த்: }\end{array}$ & ช் & $\begin{array}{l}\infty \infty \\
\dot{8} \dot{6}\end{array}$ & 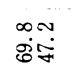 \\
\hline 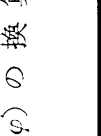 & $\stackrel{\infty}{\stackrel{\infty}{i}}$ & mº & 茫 & $\begin{array}{l}+0 \\
\text { i் }\end{array}$ & $\begin{array}{l}\dot{0} \\
\dot{8} \\
\dot{8}\end{array}$ & 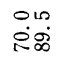 & $\begin{array}{c}+\infty \\
\substack{+\infty \\
\infty} \infty \infty \\
\infty\end{array}$ & $\begin{array}{l}\infty \\
\infty \\
\infty \dot{\infty} \\
\dot{\infty}\end{array}$ & 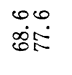 & 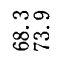 & $\begin{array}{l}\text { NN } \\
\text { ¿ุo }\end{array}$ & $\begin{array}{c}-1 \\
\infty \\
\infty\end{array}$ & $\vec{\infty}$ & 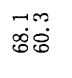 & 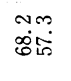 & 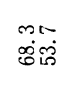 & $\begin{array}{l}\infty \\
\dot{\infty} \infty \\
\dot{0} 0 \dot{0}\end{array}$ & $\begin{array}{l}\infty \\
\infty \\
\dot{\infty} \tilde{y}\end{array}$ & $\begin{array}{l}-\tilde{N} \\
\dot{8} \dot{b}\end{array}$ \\
\hline 8 & $\stackrel{0}{i}$ & $\begin{array}{l}\text { 여 } \\
\text { î. }\end{array}$ & $\begin{array}{l}\text { om } \\
\text { تंब्త్త }\end{array}$ & 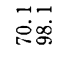 & $\begin{array}{l}\infty \\
\dot{\delta} \\
\dot{8}\end{array}$ & 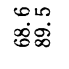 & 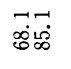 & $\begin{array}{l}100 \\
\dot{0} \\
\dot{0}\end{array}$ & $\begin{array}{l}\text { No } \\
\text { sion }\end{array}$ & 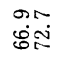 & $\begin{array}{l}\infty \infty \\
\dot{8} \infty \\
\dot{8} 0\end{array}$ & $\begin{array}{l}\infty N \\
\dot{8}: 0 \\
\dot{8}\end{array}$ & $\begin{array}{l}\infty 1 \\
\dot{8} \\
\dot{8}\end{array}$ & $\begin{array}{l}0.4 \\
\dot{0} \\
\dot{y}\end{array}$ & 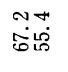 & 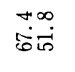 & $\begin{array}{l}\infty r \\
\dot{\delta} \dot{\alpha}\end{array}$ & $\begin{array}{c}-\infty \\
\infty \\
\infty \\
\infty\end{array}$ & 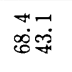 \\
\hline UV & $\stackrel{+}{\leftrightarrow}$ & $\begin{array}{l}\text { or } \\
\text { si: }\end{array}$ & 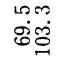 & $\begin{array}{l}\infty \infty \\
\infty 8 \\
\infty \\
\infty\end{array}$ & $\begin{array}{l}\dot{\overrightarrow{0}} \\
\dot{\vec{\theta}}\end{array}$ & 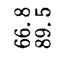 & 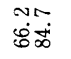 & 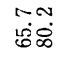 & 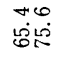 & $\begin{array}{l}3 \pi \\
\dot{8} \\
\dot{B}\end{array}$ & 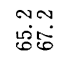 & 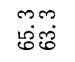 & $\begin{array}{l}+\infty \\
\text { : } \\
\text { :io }\end{array}$ & $\begin{array}{l}\text { rem } \\
\text { äcio }\end{array}$ & 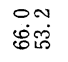 & $\begin{array}{l}\text { mo } \\
\dot{8} \% \dot{q}\end{array}$ & $\begin{array}{l}\infty+1 \\
\dot{8} \dot{y}\end{array}$ & 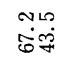 & ث். \\
\hline & i & 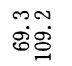 & 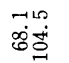 & $\begin{array}{l}00 \\
\text { ப்க் }\end{array}$ & $\begin{array}{l}010 \\
\dot{8} \dot{\circ}\end{array}$ & 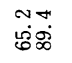 & 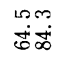 & $\begin{array}{l}\infty \infty \\
\text { ifi }\end{array}$ & 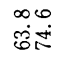 & 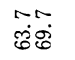 & $\begin{array}{l}\infty+\pi \\
\dot{க ் ச ி ~}\end{array}$ & $\begin{array}{l}\text { कm } \\
\text { हंढ }\end{array}$ & 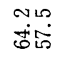 & $\begin{array}{l}\text { no } \\
\text { षं山ें }\end{array}$ & 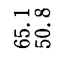 & $\begin{array}{l}\forall 0 \\
\dot{8} \theta \dot{q}\end{array}$ & $\begin{array}{l}\sigma \infty \\
\dot{b} \dot{\beta}\end{array}$ & $\begin{array}{l}+0 \\
\dot{8}=\dot{F}\end{array}$ & $\begin{array}{l}\dot{0} \\
\dot{\varphi} \dot{\infty}\end{array}$ \\
\hline & 0 & No? & No & No & $\infty$. & $\ddot{+\infty}$ & $\therefore$ & 9 & ก⿻ & $\infty$ ? & om & No. & -o. & NA & & $\infty$ & ON. & $-m$ & $\sim \infty$ \\
\hline 폭 & i & 6. & 岁哿 & 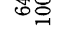 & சठ & రுం & ర్రణ & $6 \%$ & 6त्र & $6 \widehat{6}$ & ช்่ & 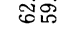 & ชู่น์ & ติเ & \%ं。 & ఫீ & 807 & bi & 8 \\
\hline & $\stackrel{\infty}{-}$ & 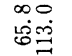 & 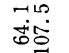 & $\begin{array}{l}00 \\
\dot{S} \dot{\sigma} \dot{⿹}\end{array}$ & $\begin{array}{l}+10 \\
\dot{6} 480\end{array}$ & 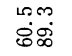 & $\begin{array}{l}\infty 0 \\
\dot{\infty} \infty \\
\dot{m} \infty\end{array}$ & 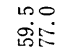 & 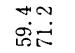 & 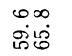 & $\begin{array}{l}\text { or } \\
\text { si̊ }\end{array}$ & $\begin{array}{l}n m \\
\text { sio } \\
\dot{s i n}\end{array}$ & 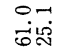 & 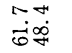 & $\begin{array}{l}\text { mo } \\
\text { त் }\end{array}$ & $\begin{array}{l}N \text { N } \\
\dot{y} \vec{b}\end{array}$ & $\begin{array}{l}\text { ON } \\
\text { tio }\end{array}$ & $\begin{array}{l}\infty \\
\dot{0} \cdot \dot{m}\end{array}$ & $\begin{array}{l}0 . \\
\text { : }\end{array}$ \\
\hline & $\stackrel{\circ}{-}$ & 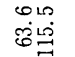 & 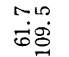 & $\dot{\overrightarrow{8}}$ & $\begin{array}{l}60 \\
\infty 08 \\
0 \\
0\end{array}$ & 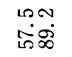 & 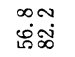 & 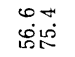 & $\begin{array}{l}\text { ro } \\
\text { ஸ் }\end{array}$ & $\begin{array}{l}N= \\
\text { iñ. }\end{array}$ & 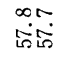 & 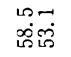 & 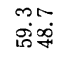 & $\begin{array}{l}\text { No } \\
\text { घं山ें }\end{array}$ & $\dot{\omega}=$ & 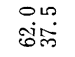 & ت0 & 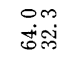 & $\begin{array}{l}\infty \sigma \\
\text { tig }\end{array}$ \\
\hline \multirow[t]{7}{*}{ W } & & $\overrightarrow{0}+\infty$ & $\begin{array}{l}\infty, \vec{r} \\
\infty \\
\infty\end{array}$ & $\begin{aligned} \sim \infty \\
\dot{0}+\end{aligned}$ & $\overrightarrow{5}$ & $\circ-1$ & $\stackrel{N-1}{n}$ & $\sim カ$ & $\sim$ & $+\infty$ & $\forall+\infty$ & $\forall m$ & 100 & $\mathscr{W N}$ & $\sim 0$ & $\because \infty$ & th & No & $\sim \infty$ \\
\hline & & & $=$ & & & की & గึळ & 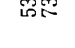 & iூת & ํำ: & 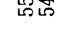 & 造\% & & 哭子 & 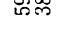 & कुले & के & $\delta_{0}$ & $8 \mathrm{~N}$ \\
\hline & $\stackrel{\sim}{\sim}$ & & 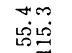 & $\begin{array}{l}\text { on } \\
\text { îं }\end{array}$ & $\begin{array}{l}\text { कि } \\
\text { inso }\end{array}$ & $\begin{array}{l}\sim \infty \\
\dot{F} \infty \infty\end{array}$ & $\begin{array}{l}\text { ஸै० } \\
\text { अंक }\end{array}$ & $\begin{array}{l}+\infty \\
\dot{q}\end{array}$ & 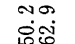 & mo & $\begin{array}{l}\infty \circ \\
\text { i. } \\
\text { i. }\end{array}$ & $\begin{array}{l}N_{0} \\
\dot{i} \rightarrow \dot{ }\end{array}$ & $\begin{array}{l}00 \\
1 \dot{0} 0\end{array}$ & $\begin{array}{l}\dot{0} \\
\dot{r i g}\end{array}$ & ம் & $\begin{array}{l}-10 \\
00 \\
00\end{array}$ & $N$ & $\begin{array}{l}\text { in } \\
\text { sing }\end{array}$ & $\ddot{\theta}$ \\
\hline & & 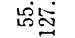 & ம் & $\stackrel{\infty}{\sigma} \doteq$ & ட்க் & 我 & $\begin{array}{ll}N 0 \\
j i N\end{array}$ & 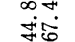 & $\begin{array}{l}\text { mo } \\
\text { gos } \\
\text { gos }\end{array}$ & $\begin{array}{l}00 \\
\dot{\alpha} \dot{q} \vec{b}\end{array}$ & 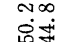 & $\dot{\pi}$ & ஸெ & 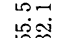 & نें & $\begin{array}{l}\infty-1 \\
\infty \\
\infty \\
\end{array}$ & ne & $\begin{array}{l}\infty 00 \\
-1\end{array}$ & $\begin{array}{l}\infty \infty \\
\dot{\infty} \sigma\end{array}$ \\
\hline & & int & om & 000 & $M$ & Nm & & & & & & & & 00 & & $\infty 0$ & & & \\
\hline & 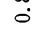 & ம் & 字桪 & ช่ذ & हिंత్తి & $\dot{m} \infty \dot{\infty}$ & ळंホ & க்่ & ซ்i & 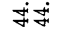 & $\dot{\sigma} \ddot{\circ}$ & ச் & ஸ่ંั & मீंब & ம்வ & कितें & ச்ற் & $\dot{\theta}=\dot{\pi}$ & ช્ \\
\hline & $\stackrel{\circ}{\circ}$ & $\begin{array}{l}\infty, 9 \\
\dot{\forall} \vec{\exists}\end{array}$ & 可. & $\begin{array}{l}0 n \\
\dot{m} \overrightarrow{\mathrm{N}}\end{array}$ & $\begin{array}{l}+\infty \\
\dot{m} \dot{\varnothing}\end{array}$ & 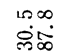 & $\begin{array}{l}\text { No } \\
\text { ت. }\end{array}$ & $\begin{array}{l}\infty m \\
\text { min }\end{array}$ & 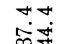 & $\begin{array}{l}-10 \\
\Rightarrow \dot{\forall}\end{array}$ & $\begin{array}{l}\text { मnt } \\
\dot{y}=\dot{0}\end{array}$ & $\begin{array}{l}010 \\
0.08\end{array}$ & జN & $\begin{array}{l}\infty 0 \\
\text { is }\end{array}$ & $\begin{array}{l}\sigma 00 \\
\text { tis }\end{array}$ & $\begin{array}{l}-10 \\
\dot{n}=0\end{array}$ & $\begin{array}{l}\text { or } \\
\text { gif }\end{array}$ & $\ddot{\Delta}$ & $\overrightarrow{\text { ธิن }}$ \\
\hline \multirow{3}{*}{$\begin{array}{l}0 \\
\stackrel{0}{0} \\
0 \\
0\end{array}$} & & $\stackrel{+*}{+}$ & No & $\infty \infty$ & 6in & +0 & $\infty$. & $+\infty$ & $\Rightarrow 7$ & om & $m$ & $\theta+$ & 00 & $\infty 0$. & $-\infty$ & no & $\infty \pi$ & mo. & D. \\
\hline & & & $m$ & जm & $N=$ & సळ & No & $\vec{m} \dot{q}$ & men & & & & & & & & & & \\
\hline & $\stackrel{\sim}{0}$ & $\begin{array}{l}9 m \\
\dot{\forall}: 0 \\
\dot{g}\end{array}$ & $\begin{array}{l}100 \\
\text { rib }\end{array}$ & 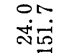 & 영 & $\begin{array}{l}N m \\
\exists \infty \\
\exists \infty\end{array}$ & 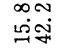 & $\begin{array}{l}\text { 수 } \\
\text { ฌंकें }\end{array}$ & 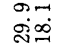 & $\begin{array}{l}\infty \infty \\
\dot{m} \tilde{m}\end{array}$ & 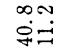 & $\begin{array}{l}\infty \neq \\
\dot{j} \sigma\end{array}$ & 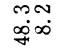 & $\vec{B}$ & 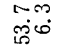 & $\begin{array}{l}\text { NLn } \\
\text { Nibn }\end{array}$ & $\begin{array}{l}m 0 \\
\infty \\
\infty \\
\infty\end{array}$ & $\overrightarrow{8}^{-10}+8$ & $\overrightarrow{\dot{b}}=\overrightarrow{+}$ \\
\hline i & $\stackrel{\circ}{\circ}$ & $\stackrel{\circ}{\vec{\forall} \infty}$ & $\begin{array}{l}\infty \\
\dot{m} \otimes \infty\end{array}$ & تُ & $\stackrel{0}{\dot{\oplus} \infty}$ & $\stackrel{m}{-i 0}$ & $\stackrel{\infty}{=}$ & ஜ் & న్ & : & No & I0 & 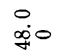 & i் & ஸ் & ت্ं0 & $\infty_{\infty}^{\infty}$ & $\stackrel{0}{\circ 0}$ & $\stackrel{0}{-i 0}$ \\
\hline \multirow[t]{2}{*}{$\widehat{\nabla a}$} & & & & & & & & & & & & & & & & & 0 & & \\
\hline & & & i & 0 & $\because$ & $\approx$ & 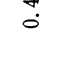 & : & $\begin{array}{l}\infty \\
0 \\
0\end{array}$ & - & $\stackrel{2}{-}$ & $\stackrel{-}{-}$ & $\stackrel{\circ}{-}$ & $\stackrel{\infty}{-}$ & $\dot{\sim}$ & ה & i & i & $m$ \\
\hline
\end{tabular}




\begin{tabular}{|c|c|c|c|c|c|c|c|c|c|c|c|c|c|c|c|c|c|c|c|}
\hline 毁 & $\stackrel{0}{\dot{0}}$ & $\begin{array}{l}+\infty \\
\text { జ் }\end{array}$ & $\begin{array}{l}\text { के } \\
\dot{\infty} \phi\end{array}$ & $\begin{array}{l}+\infty \\
\dot{\infty}+\dot{\infty} \\
\dot{m}\end{array}$ & 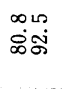 & $\begin{array}{l}\text { சீं } \\
\text { ळ் }\end{array}$ & $\begin{array}{l}\sigma \infty \\
\dot{1} \infty \\
\dot{1} \infty\end{array}$ & $\begin{array}{l}+\infty \\
\text { تి }\end{array}$ & 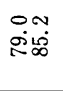 & $\begin{array}{l}\infty 00 \\
\infty \\
\infty \\
\infty\end{array}$ & 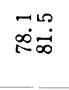 & مَ & : & 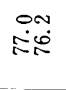 & $\begin{array}{l}\dot{0} \\
\dot{Q} \dot{N}\end{array}$ & $\begin{array}{l}\text { Nm } \\
\dot{E}\end{array}$ & $\begin{array}{l}\text { on } \\
\text { gis. }\end{array}$ & 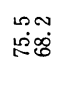 & $\begin{array}{l}-\infty \\
\dot{8}: 8\end{array}$ \\
\hline 新 & $\stackrel{2}{6}$ & 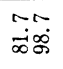 & $\vec{\infty}$ & $\dot{0}$ & $\begin{array}{l}\ddot{\sim} \\
\dot{\infty} \tilde{\Phi}\end{array}$ & sis & 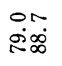 & $\begin{array}{l}\infty \\
\infty \\
\infty\end{array}$ & 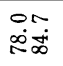 & $\begin{array}{l}\infty \\
\stackrel{\infty}{*}\end{array}$ & त्. & $\begin{array}{l}-\infty \\
0 \infty\end{array}$ & $\begin{array}{l}N \infty \infty \\
\substack{\infty \\
0.0}\end{array}$ & $\begin{array}{l}90 \\
\text { Siti }\end{array}$ & 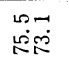 & 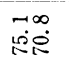 & 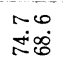 & 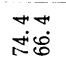 & 证 \\
\hline & is & $\begin{array}{l}\text { :u } \\
\dot{\infty}\end{array}$ & 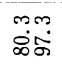 & sis & 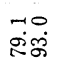 & $\begin{array}{l}n \infty \infty \\
\infty \\
\infty \\
\infty\end{array}$ & 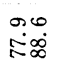 & 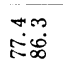 & $\begin{array}{l}\sigma \mathcal{N} \\
\dot{0}\end{array}$ & $\begin{array}{l}+09 \\
\dot{Q}-\vec{\infty}\end{array}$ & $\begin{array}{l}\sigma_{\infty}^{\infty} \\
\text { nisi }\end{array}$ & 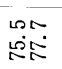 & $\begin{array}{l}060 \\
\text { sis. }\end{array}$ & $\begin{array}{l}\text { Oun } \\
\text { İpo }\end{array}$ & 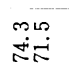 & लंक्ष & 80 & ק̇ं & $\begin{array}{l}\text { ã } \\
\text { iิ }\end{array}$ \\
\hline & $\stackrel{\text { L }}{+}$ & $\begin{array}{l}010 \\
\dot{8} \\
\dot{8}\end{array}$ & $\begin{array}{l}m \sim \\
\text { Pio }\end{array}$ & 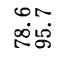 & $\begin{array}{l}\text { शm } \\
\text { im }\end{array}$ & $\begin{array}{l}m \infty \\
\text { i̊ } \\
\text { is }\end{array}$ & $\begin{array}{l}\dot{0} \\
\dot{\infty} \infty \\
\dot{\infty} \infty\end{array}$ & 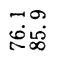 & مُ & 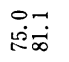 & 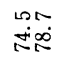 & $\begin{array}{l}\text { Om } \\
\text { +iष }\end{array}$ & :-7 & हై & $\begin{array}{l}90 \\
\text { הి. }\end{array}$ & $\begin{array}{l}n 0 \\
\text { îंष }\end{array}$ & 点峞 & $\begin{array}{l}\infty 0 \% \\
\vec{i} \cdot \overrightarrow{0}\end{array}$ & $\begin{array}{l}\text { no } \\
\text { iٓ }\end{array}$ \\
\hline & $\stackrel{\circ}{+}$ & $\begin{array}{l}\infty \infty \infty \\
\text { o. } \\
\infty\end{array}$ & $\begin{array}{l}\text { on } \\
\text { वंg }\end{array}$ & 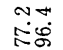 & 8 & ர். & 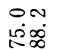 & 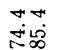 & జే. & $\begin{array}{l}\text { No } \\
\text { nंक्ठ }\end{array}$ & $\begin{array}{l}\text { mom } \\
\text { in: }\end{array}$ & $\begin{array}{l}m_{\infty}^{\infty} \\
\text { ił }\end{array}$ & \begin{tabular}{l}
$\infty \times N$ \\
\hdashline$i N i$
\end{tabular} & ic & ت- & $\begin{array}{l}\infty 18 \\
0.0 \\
0.0\end{array}$ & $\stackrel{+r}{:}$ & $\begin{array}{lll}n-1 \\
20.0\end{array}$ & $\begin{array}{l}00 \\
i \oplus \dot{0}\end{array}$ \\
\hline & & -6 & $\Upsilon^{\infty}$ & $\begin{array}{ll}+1 \\
0\end{array}$ & 60 & $\infty$. & & & $\infty N$ & & -10 & & $\infty-1$ & L 1010 & $\rightarrow \infty$ & $\infty \pi$ & & & NO \\
\hline & $\tilde{m}$ & 赵令 & న\& & 208 & ஜீガ & ரீळ & 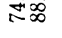 & พேை & ลิષ & iㅛ & +8 & ¿ॄ. & Rे & iø & 2i⿱宀⿻心㇒日 & ச்่ & 8:ं & 8i: & मृं \\
\hline & 8 & R. & نे & 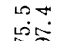 & $\begin{array}{l}\mathscr{0} N \\
\dot{T}+\dot{T}\end{array}$ & $\begin{array}{ll}\infty & =7 \\
m & =0\end{array}$ & $\dot{m}$ & $\underset{\substack{\infty \\
i \rightarrow i}}{ }$ & $\stackrel{01}{1}$ & $\begin{array}{r}-10 \\
\rightarrow \infty\end{array}$ & 100 & -6 & $\sim^{\infty}$ & $m=$ & -10 & $\infty$ & $0 \not$ & $+\infty$ & mo \\
\hline & $\dot{m}$ & & & & & & & & $i-\infty$ & $\pi N$ & & $\therefore i$ & б. & 186 & 80 & 06 & dis & 象通 & $8 \%$ \\
\hline & 虫 & id & బ. & $\begin{array}{l}+\infty \\
\dot{5} 5\end{array}$ & \begin{tabular}{l}
$\infty 00$ \\
\hdashline .5
\end{tabular} & $\begin{array}{l}\mathscr{0} \\
\dot{v} \\
\dot{v}\end{array}$ & $\begin{array}{l}\infty \infty \\
-\infty\end{array}$ & تُ & $\begin{array}{ll}+0 \\
0-\end{array}$ & $\begin{array}{ll}\infty \\
\infty i n\end{array}$ & 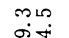 & $\begin{array}{l}\infty-\pi \\
\infty\end{array}$ & $\log _{\infty}^{\infty}$ & No & gr & $\because \div 3$ & เ? & $\forall 0$ & $\forall a$ \\
\hline 惟 & & & & & & & & & & & & & $0 \%$ & 잉명 & 60 & 68 & 8 & ธ。์ & 66 \\
\hline 虹木 & $\begin{array}{l}\dot{0} \\
\dot{r r}\end{array}$ & $\begin{array}{l}\forall 06 \\
\dot{20}\end{array}$ & $\begin{array}{l}m-1 \\
\end{array}$ & mo & $\begin{array}{l}\text { No } \\
\text { NiLj }\end{array}$ & $m \rightarrow$ & 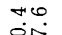 & \%o & sm & $m r$ & $\infty 0$ & no & $-\infty$ & $\infty \infty$. & $\infty \infty$ & $\forall \leftarrow$ ? & $+\infty$ & 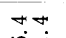 & +2 \\
\hline 莎 & & & & & & & & 800 & $\infty \infty$ & or & 60 & $6 \pi$ & 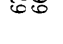 & 0 & 88 & oin & \& & $8 \pi$ & $8 \%$ \\
\hline 6 & $\stackrel{\infty}{\infty}$ & $\begin{array}{l}10 \pi \\
+\ddot{\theta}\end{array}$ & $m 0$ & $\overrightarrow{\mid r N}$ & 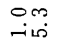 & $\circ$ & $\exists$ & $m$ & $\mathscr{O}$ & $\infty \infty$ & Lก & $\rightarrow$ & am & $\sim N$ & מ.? & 100. & เ2 & เి & $\simeq$ \\
\hline Q. & & & & & & रक & $b_{\infty}^{\infty}$ & $\infty \infty$ & $6 \pi$ & 60 & $8 \pi$ & 80 & 녕 & 180 & 888 & 링요 & 묭ำ & 명 & பில் \\
\hline$\dot{s}$ & $\stackrel{\bullet}{i}$ & 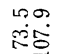 & $\begin{array}{l}\text { No } \\
\text { Nm}\end{array}$ & $\begin{array}{l}\infty \infty \\
\infty \infty \\
\infty\end{array}$ & sis & $\because 0$ & 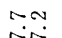 & $\infty 0$ & $-\infty$ & $+\infty$ & $\rightarrow 0$ & $\infty \sim$ & Lon & $\forall \rightleftarrows$ & $\forall m$ & +0 & เి. & om & $\infty$. \\
\hline$-v$ & & & 10 & 15 & कळ & ๘ం & $6 \infty$ & 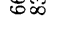 & 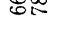 & 岁丈 & B⿺辶 & 80 & వ్రి & \$8 & tơ & ธึ่ & सें० & 8 & சீं \\
\hline$\hat{\gamma}$ & $\stackrel{\vec{\alpha}}{\sim}$ & $\begin{array}{l}\text { mm } \\
\text { ioj. }\end{array}$ & $\begin{array}{l}\text { Oo } \\
\text { Silo }\end{array}$ & 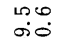 & $\begin{array}{l}x-1 \\
\infty i c\end{array}$ & 00 & $\because 0$ & $\sim *$ & no & 90. & $\log$ & m. & $\sim \infty$. & 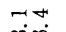 & $\because \neg$ & Nオ & & 10 & \\
\hline & $\sim$ & 10 & 중 & O. & ๘ळ & Б5 & $\ddot{\infty}$ & రాలు & ఫึะ & $8 \pi$ & 88 & 88 & 80 & గి心 & ชீடீ & छิ & லீஷ & ச்டி & เేరิ \\
\hline 6 & $\approx$ & $\begin{array}{l}0 \infty \\
-i 0 \\
-i 0\end{array}$ & $\ddot{m}$ & 90 & $\because$ & $\because 1$ & $\rightarrow$ & $m$ & $+\infty$ & $\because N$ & $\infty$. & 00 & 6 & 6.7 & & 00 . & $m 0$ & $\sim \infty$ & $-\infty$. \\
\hline 6 & $N$ & $\therefore=$ & 임 & 馬 & $8 \%$ & ஜேே & రాల & $\ddot{8} \vec{\infty}$ & 8\% & ภู่ & 50 & 68 & छम & छं & ธ่ં & તિં & வ்டி & ช่ำ & ஐீळి \\
\hline 5 & 0 & & $\infty \infty$. & or & $+\infty$ & $\infty^{\infty}$. & S. & 00. & $+\infty$. & 0,0 & $\infty \infty$ & & om & Nֵ? & $\forall$ & $\infty$. & $m a$. & & $\sim N$ \\
\hline & i & 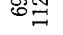 & 跑 & 8 & 85 & ஜே & $5 \infty$ & 68 & 82 & 88 & मேं & में & 8 in & 8ீ & 8. & தி & சंभ & பंలె & वृष्ले \\
\hline & $\infty$ & 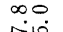 & $\infty$ & $\infty=$ & & 10-7. & & 100 ? & $\sigma .7$ & $\because$ & Yn & - & & & & LN & $\sim \infty$ & $\infty \sigma$ & $\forall \infty$ \\
\hline is & $-i$ & 6 & 녕용 & ஜ்ర్త & 1ీ & ৪ి & ஸि॰ & אי & 的㐫 & $\sin 0$ & ஸ் & în & மூர் & ம் & की & ம்று & த் & $\dot{8} \dot{0}$ & बिं \\
\hline & $\stackrel{0}{-}$ & $\begin{array}{ll}\infty \\
\dot{\theta}\end{array}$ & $\begin{array}{l}100 \\
\text { జై }\end{array}$ & № & $\begin{array}{l}N N \\
\sigma \infty\end{array}$ & $\begin{array}{l}6 m \\
\text { rnd }\end{array}$ & m. & no & $\because \approx$ & 00 & No & 00 & $\because \sim$ & $\sim \mathcal{Y}$ & $\forall r$ & $\sim \infty$. & 00 & or & ON \\
\hline & & & & & & & & & & & & & & & & & मूल & हैं & कह \\
\hline & $\stackrel{\square}{-}$ & تే: & $\dot{x}$ & $\rightarrow \infty$ & 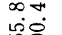 & $\begin{array}{l}\text { on } \\
+i n\end{array}$ & $\sim^{\infty}$ & on? & $\infty \circ$ & ㄱ. & น? & No & $0 .+$ & 0. & $\infty$ & \begin{tabular}{l}
$\infty$ \\
$\infty$ \\
\hdashline
\end{tabular} & $\theta=$ & $\cdots$ & $\infty$ \\
\hline & & I & $=$ & & & & & & & & & L & ம்\% & เ人⿱一𫝀口十 & मुल & & โم: & దిల్లి & iిం \\
\hline & $\stackrel{\sim}{\sim}$ & 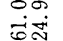 & $\underset{\sim \infty}{\sim \infty}$ & $\begin{array}{l}100 \\
4: 0\end{array}$ & $\begin{array}{l}\infty N N \\
-\sim \pi\end{array}$ & $\overrightarrow{\sigma m p}$ & $\begin{array}{l}\infty, 0 \\
\infty \infty m\end{array}$ & S. & $\begin{array}{l}-\infty \\
\infty \\
\infty\end{array}$ & $\begin{array}{l}\text { జक } \\
\infty \infty \infty\end{array}$ & $\mathscr{0 N}$ & $\therefore$ & 90 & $\Rightarrow=$ & No. & 00 & $\infty-$ & $0-7$ & Oㄴ? \\
\hline & & & & & & & & & & & & & & เॉल & மா & டா, & దू⿻ & 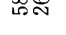 & म재 \\
\hline & & $\vec{\infty}: 0$ & $\begin{array}{l}\sim \infty \infty \\
\dot{\gamma} \sim\end{array}$ & $\begin{array}{l}N m \\
\dot{0}\end{array}$ & $\begin{array}{l}\sigma 10 \\
0 \\
0+4\end{array}$ & ON. & $m$ & Nm & $\infty \infty$ & or & ino & $\because+$ & $\infty \infty$. & ?. & $\infty \infty$. & mo & $\theta 0$. & -6 & \\
\hline & & $\stackrel{m}{m}$ & $10 \simeq$ & $n z$ & +0 & Fo' & $+\infty$ & 42 & F్ర & \&列 & 아영 & ক্বন & Fิ & மேங் & గ్రిలి & เேล & 员太 & in & దని \\
\hline & $\stackrel{\infty}{\infty}$ & $\begin{array}{l}\text { Lm } \\
\text { tid }\end{array}$ & $\begin{array}{l}N \sigma \\
\infty \infty \infty\end{array}$ & त्रक & $\stackrel{+\infty}{-i}$ & $\infty$ & $\because 9$ & $\mathscr{O N}$ & Om & $=m$ & $m 0$. & $\mathscr{O N}$ & $\because 9$ & $\mathscr{0}+7$ & mo & br & 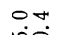 & +40 & \\
\hline & 0 & దற్తు & יర్తు & $=$ & F。् & ల్లా & న্ & 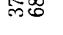 & & $F^{\infty}$ & F & \&্口 & नेल & ;ాని & డై & దN & นగదిని & 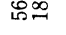 & 的旦 \\
\hline & & G+ & कीज & +0 & $\exists$ & 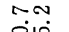 & $\infty$ & mos. & S.t. & $-\infty$ & $m$ & Nr. & $\infty 0$ & $\because \rightarrow$ & $\because$ & $\forall$ & N. & $\infty$. & \\
\hline & & $\overrightarrow{10} \pm$ & अू⿹ & మిస్త & ల్లే) & లిః & నిం & लై & ణ্ & 하유 & ;్ల & ఖా & 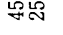 & 需 & 요 & กิ드 & 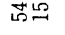 & 绐五 & โิ่า \\
\hline & & ㄴ? & $\stackrel{\infty}{\infty}$ & & mo & & $+\infty$ & om & 09. & o. & & $\because$ & & & & & 70 & & \\
\hline 늑 & $\dot{0}$ & 舟焉 & m̧⿻ & mi & ปี่ & స். & สี่ & ลีं & शి. & लंख் & $\dot{\dot{m}} \dot{\Delta}$ & 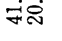 & $\dot{j}=\dot{1}$ & ச்่ & $\dot{q} \dot{m}$ & 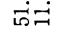 & ค่@ & คُ๐் & $\operatorname{lin}^{\infty}$ \\
\hline & & $\begin{array}{l}\infty 0 \\
0 \notin 0\end{array}$ & $+\pi$ & $\therefore \rightarrow-1$ & $\sim \infty$ & & मी. & ro. & $\cong$. & $\infty$ & & & & & & & m. & $-\infty$. & \\
\hline & $\dot{s}$ & 胥递 & 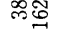 & 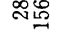 & $\stackrel{\infty}{-\infty} \underset{్}{\infty}$ & $=0$ & 고ำ & $\stackrel{\infty}{\infty} \underset{\tilde{m}}{ }$ & నిసี & எ료 & దీతి & లిల & $\dot{q}^{\infty} \infty$ & Sin & $\stackrel{g}{\sigma 0}$ & [نं & ค่̉ & மூ்่ & சீं \\
\hline 1 & $\stackrel{0}{\circ}$ & 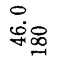 & $\stackrel{\sim}{\stackrel{\sim}{\infty} \infty}$ & 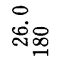 & : & $\check{\dot{m}}_{\stackrel{D}{ }}$ & $\stackrel{0}{\dot{0}}$ & 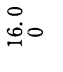 & జֶఞ & స్లెం & బై & మొ & ஷ்o & 字० & $\begin{array}{l}m \\
\infty \\
\dot{\infty} \sigma 0\end{array}$ & i० & ஜีंం & में & $\begin{array}{l}0 \\
\dot{\circ} \\
i\end{array}$ \\
\hline & & $\begin{array}{l}\dot{0} \\
\dot{0} \\
i\end{array}$ & $\begin{array}{l}\ddot{0} \\
0 \\
1\end{array}$ & $\begin{array}{l}N \\
0 \\
0 \\
1\end{array}$ & $\because$ & $\tilde{0}$ & $\stackrel{\overrightarrow{0}}{0}$ & $\stackrel{0}{\circ}$ & 恶 & $\stackrel{\circ}{i}$ & $\dddot{\sim}$ & $\stackrel{+}{-}$ & $\stackrel{\circ}{-}$ & $\stackrel{\infty}{-}$ & $\stackrel{\stackrel{i}{i}}{i}$ & 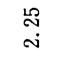 & i⿱口 & 足 & $\ddot{m}$ \\
\hline
\end{tabular}




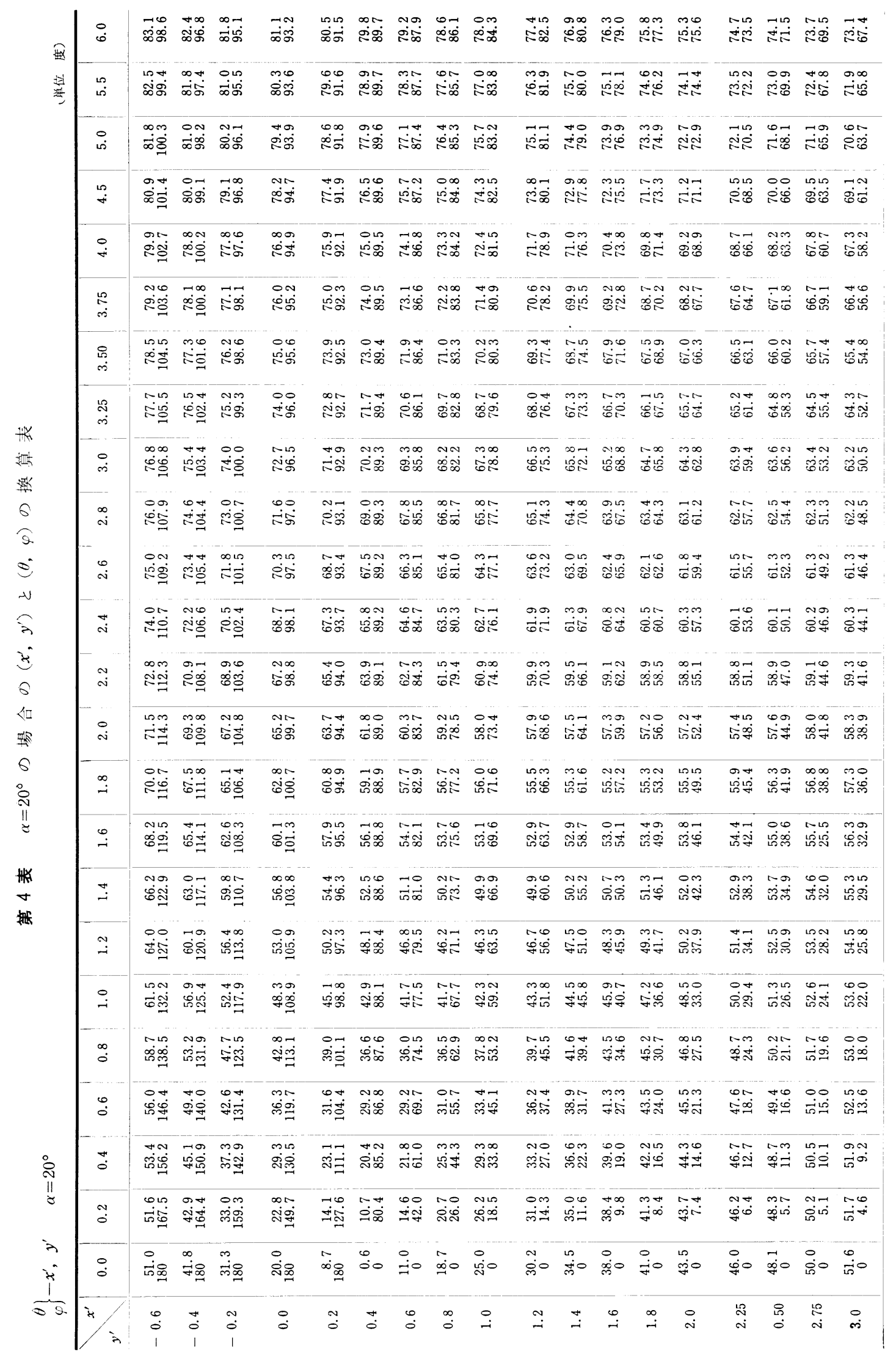




\begin{tabular}{|c|c|c|c|c|c|c|c|c|c|c|c|c|c|c|c|c|c|c|c|}
\hline 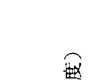 & $\dot{0}$ & $\begin{array}{l}\infty-7 \\
\infty \\
\infty\end{array}$ & 芯 & జ். & 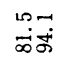 & $\begin{array}{l}\mathscr{m} \\
\dot{\infty} \\
\dot{8}\end{array}$ & $\begin{array}{l}\text { क० } \\
\text { शं8 }\end{array}$ & $\begin{array}{l}-\infty \\
\sigma^{\infty} \infty \\
\sigma_{\infty}^{\infty}\end{array}$ & & 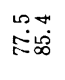 & $\begin{array}{l}\infty \\
\dot{\infty} \infty \\
\dot{\infty} \infty\end{array}$ & $\begin{array}{l}-10 \\
\dot{0}: 0\end{array}$ & $\begin{array}{l}\forall \infty \\
\text { ம்ळ }\end{array}$ & $\begin{array}{l}\infty 00 \\
\stackrel{\infty}{+\infty} \infty \\
+\infty\end{array}$ & $\begin{array}{l}\text { to } \\
\text { सie }\end{array}$ & $\begin{array}{l}\text { mo } \\
\text { miti }\end{array}$ & 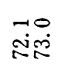 & $\begin{array}{l}00 \\
\text { is: }\end{array}$ & تंठ̈. \\
\hline 武 & L? & $\begin{array}{l}\text { mo } \\
\dot{\infty} \dot{8}\end{array}$ & $\begin{array}{l}\overrightarrow{+}+\vec{\infty} \\
\dot{\infty} \infty\end{array}$ & $\begin{array}{l}\text { mo } \\
\text { - }\end{array}$ & $\ddot{\infty} \dot{\infty}$ & ㄴ. & $\begin{array}{l}\infty 0 \\
\infty ் 8 \\
\infty\end{array}$ & ra & $\begin{array}{l}\text { mo } \\
\text { ¿\& }\end{array}$ & $\begin{array}{l}n 0 \\
\text { nid } \\
2 \infty \infty\end{array}$ & ت્ळ & 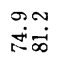 & $\begin{array}{l}\text { No } \\
\text { +io }\end{array}$ & $\begin{array}{l}00 \\
\text { min }\end{array}$ & $\begin{array}{l}\infty \infty \\
\text { ivi } \\
\text { Nis. }\end{array}$ & ్ㅗ & م: & $\begin{array}{l}0 .+ \\
\text { 용 }\end{array}$ & 웅 \\
\hline & is & ஸ̊ & $\begin{array}{l}\infty \sigma \\
\dot{\infty} \dot{\infty}\end{array}$ & $\begin{array}{l}\text { ros } \\
\dot{\infty} \& \dot{s}\end{array}$ & $\begin{array}{l}\text { iீ } \\
\text { i் }\end{array}$ & $\begin{array}{l}+\infty \\
\infty \\
\infty \\
\infty\end{array}$ & $\begin{array}{l}\text { 오 } \\
\text { 효 }\end{array}$ & $\begin{array}{l}00 \\
i \infty \infty \\
\hat{N} \infty\end{array}$ & $\begin{array}{l}\text { TL } \\
\dot{\varphi}\end{array}$ & $\begin{array}{l}\text { NLR } \\
\text { शै: }\end{array}$ & \begin{tabular}{l}
$m+$. \\
\multirow{i}{*}{$\infty$}
\end{tabular} & ஜ். & 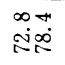 & 空送 & 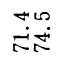 & 옹- & $\begin{array}{l}\infty \infty \\
\text { வீंే }\end{array}$ & تص: & $\begin{array}{l}\overrightarrow{8} \\
\dot{0}: 0\end{array}$ \\
\hline & $\stackrel{4}{+\infty}$ & 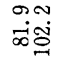 & $\begin{array}{l}\infty \sigma \\
\dot{\infty} \dot{\delta}\end{array}$ & $\begin{array}{l}\text { ro } \\
\text { iूं }\end{array}$ & 悉官 & 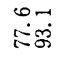 & $\begin{array}{l}0 \infty \\
\dot{0} 8\end{array}$ & 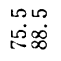 & 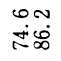 & ro & 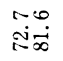 & 穴 & 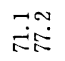 & $\begin{array}{l}\text { mo } \\
\text { 용 }\end{array}$ & $\begin{array}{l}00 \\
\text { వై }\end{array}$ & $\begin{array}{l}\infty m \\
\infty \\
0.0\end{array}$ & क्ष & $\begin{array}{l}92 \\
808 \\
808\end{array}$ & ت̊. \\
\hline & $\stackrel{\circ}{+}$ & $\begin{array}{l}00 \\
\dot{\infty} \dot{g} \\
\dot{g}\end{array}$ & & $\begin{array}{l}100 \\
\infty \\
\infty \\
\infty\end{array}$ & $\begin{array}{l}3-\overrightarrow{8} \\
\stackrel{2}{8}\end{array}$ & 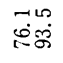 & $\begin{array}{l}0 \% \\
\text { ம்த் }\end{array}$ & $\begin{array}{l}\infty \infty \\
\prod_{\infty}^{\infty} \infty\end{array}$ & 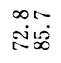 & $\vec{i}$ & $\begin{array}{l}\infty 0 \\
\stackrel{\infty}{\infty} \dot{0}\end{array}$ & $\begin{array}{l}00 \\
80 \\
00\end{array}$ & $\begin{array}{l}00 \\
\text { gi: }\end{array}$ & 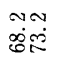 & $\begin{array}{l}\operatorname{lng} \\
\text { 용 }\end{array}$ & $\begin{array}{l}\text { 웅 } \\
\dot{8}: 0\end{array}$ & $\begin{array}{l}\text { சm } \\
\dot{800}\end{array}$ & $\begin{array}{l}m \\
\text { ติ่ }\end{array}$ & 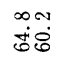 \\
\hline & 10 & $\begin{array}{l}\forall \vec{\infty} \\
\dot{\infty}\end{array}$ & 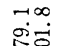 & $\begin{array}{l}\infty-1 \\
\therefore \sigma\end{array}$ & ن. & $\begin{array}{l}N 1 \\
10 \%\end{array}$ & iog & 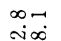 & J゙ & $\begin{array}{l}100 \\
001\end{array}$ & 00 & $\because$ & $\infty \sim N$ & $\rightarrow n$ & $m$ & เงเ & $\sigma_{\infty}^{\infty}$ & $?$ & 70 \\
\hline & $\dot{m}$ & & & & & & & & & & $0 \infty$ & ON & 62 & 60 & 08 & 08 & 80 & 56 & ஜx \\
\hline & 요 & के. & $\begin{array}{l}\forall 0 \\
\infty 0 i\end{array}$ & $\begin{array}{l}0 \infty \\
\because \infty\end{array}$ & $\begin{array}{l}100 \\
100\end{array}$ & $m o$ & $\begin{array}{l}\text { so } \\
\text { if }\end{array}$ & To. & 207 & ?. & $\forall$ & $\forall$ & $\infty 0$ & เ?. & $\because$ & $m \sim$ & o? & $0 . t$ & $n \infty$. \\
\hline & $\dot{r}$ & & $\approx 0$ & & டーの & & तே & $\therefore \infty$ & $8 \infty_{0}$ & 800 & so & 50 & $8 \approx$ & $8 \pi$ & 40 & $58: 8$ & ชิ & గిం & ชิ요 \\
\hline & $\stackrel{\leftrightarrow}{\leftrightarrow}$ & $\begin{array}{l}-0 \\
\text { Sig }\end{array}$ & $\begin{array}{l}\infty \\
\therefore \otimes\end{array}$ & $\begin{array}{l}-0 \\
\dot{0}\end{array}$ & In: & $\overrightarrow{r+N}$ & :. & $\because \pi$ & $\vec{\sigma} \overrightarrow{0}$ & $\begin{array}{ll}0 & 0 \\
\infty & 0\end{array}$ & $\begin{array}{l}9+ \\
\dot{\infty}\end{array}$ & 0. & $\vec{\Delta}$ & $m 0$ & 00 & 0.0 & ro & ris & $m \infty$ \\
\hline 满 & 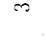 & & & & & & & & $6 \infty$ & & Dot & o & & 60 & & 38 & 10.0 & 응 & $\overrightarrow{6}$ \\
\hline 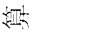 & $\stackrel{0}{\dot{r}}$ & $\begin{array}{l}m \infty \\
\infty \\
\infty \\
v-1\end{array}$ & $\begin{array}{l}6 \\
\dot{0}\end{array}$ & ம்- & $\begin{array}{r}\forall \\
\dot{0} \infty\end{array}$ & $\stackrel{\infty}{-\infty}$ & $\ddot{0}$ & or & $\stackrel{\mathscr{N}}{\sim}$ & +0 & mL & $+\infty$ & 10.7 & $\overrightarrow{r a x}$ & $\overrightarrow{r N}$ & +2 & o. & $\because \square$ & $\because 0$ \\
\hline 雨 & & & & & & & & & $0 \infty$ & & on & So & on & 60 & 80 & 60 & 820 & D. & 6 in \\
\hline 6 & $\stackrel{\infty}{\infty}$ & $\begin{array}{l}00 \\
\therefore 8\end{array}$ & nis & $\begin{array}{l}0 \tilde{N} \\
\dot{x} \dot{y}\end{array}$ & $\begin{array}{l}\infty \infty \\
i \infty\end{array}$ & $\begin{array}{l}00 \\
\dot{0}: 0\end{array}$ & $\begin{array}{l}0 \sim \\
\dot{S}-1\end{array}$ & $\begin{array}{ll}102 \\
\therefore \approx\end{array}$ & $\begin{array}{l}\sim \infty \\
i \infty\end{array}$ & $\begin{array}{l}\text { gN } \\
\dot{H}\end{array}$ & $\begin{array}{l}\text { ox } \\
\text { riso }\end{array}$ & av & $\begin{array}{l}\text { ro } \\
\text { io }\end{array}$ & $\begin{array}{l}\longrightarrow 0 \\
-0.0\end{array}$ & $\because 0$ & To & $\because$ & mis & or \\
\hline S. & & & & & & & & & & & & & & & 60 & 80 & D̆ & bis & iم: \\
\hline$\theta$ & $\stackrel{0}{\sim}$ & $\begin{array}{l}\infty \pi \\
\dot{0}\end{array}$ & $\begin{array}{l}\infty \infty \\
\dot{x}: 0 .\end{array}$ & बि. & $O N$ & $\sim \nabla$ & Lִ ?ִ & $\because$ & $\theta \overrightarrow{.}$ & mo & 31 & $\because 0$ & เמ? & $\infty$ & $3 \sigma$ & $\infty$ & $\because$ & 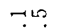 & o.m \\
\hline 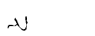 & & $\cdots=$ & 19 & to & & 6 & 65 & 000 & $8 \infty$ & 60 & OST & $\overrightarrow{6} \mathbb{1}$ & 808 & is & Bम & 冓恶 & 号じ & ஸீచ & 的京 \\
\hline & $\forall$ & $\begin{array}{l}\infty \sigma \\
\dot{2}=\end{array}$ & $\overrightarrow{m \infty}$ & ت-7. & $\stackrel{\infty}{\infty}$ & $\because$ & $\because 4$ & $\mathrm{~N}$. & $\sim \infty$ & 00 & $+\infty$ & 106 & $\infty$ ? & $\sim \sim$ & $\because 0$ & $?$ & 0.0 & $\sigma \curvearrowright$ & $\infty \mathrm{m}$ \\
\hline & N & & $\therefore=$ & Fo & హ) & 68 & & $4 \infty$ & ర్రిం్ & $\overrightarrow{6}: 0$ & $8 \pi$ & inie & 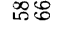 & 18 & 508 & 鞄通 & 的芯 & 品急 & 舟尔 \\
\hline & ๆ. & $\begin{array}{l}\infty r \\
\dot{\forall}\end{array}$ & $\begin{array}{l}\text { Lit } \\
\text { io }\end{array}$ & ヘ? & $\begin{array}{ll}0: 0 \\
\infty\end{array}$ & sm & 90. & $m \infty$ & $\because N$ & no & $\forall \sim$ & 09 & 00 & เว. & or & $\infty n$ & $0-1$ & on & $-\infty$. \\
\hline 6 & 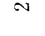 & $\therefore=$ & ¿0 & $\therefore$ & & பீ) & சே & తిథ & 80 & s: & 冓 & {$\left[\begin{array}{l}\infty \\
\infty\end{array}\right.$} & 158 & 100 & 范旗 & 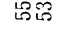 & 108 & 绐子 & 䠌等 \\
\hline & 0 & $\begin{array}{l}\infty \infty \\
\text { mis }\end{array}$ & $ت$ & $\begin{array}{l}L \infty \infty \\
\infty 00^{\circ}\end{array}$ & - & ros & $\because$ & תמו & $\forall$ & $\Upsilon$ & ט. & $\forall \multimap$ & $10 \infty$ & $10 \infty$ & $m$ & ฯ. & ヘ? & $\because 0$ & 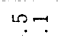 \\
\hline 艮 & & $\exists$ & $=$ & 00 & & 13\% & $\overrightarrow{\nabla ే}$ & గిळ & $\infty ீ \dot{\infty}$ & ธุ์ & దீЕ & 1050 & ธี่ & 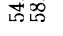 & ธี่เి & मேं & เึ่字 & 它 & Б゙F \\
\hline is & $\stackrel{\infty}{-}$ & $\begin{array}{l}m \sim \\
i \infty \infty\end{array}$ & $\begin{array}{l}\forall 00 \\
8 \infty \infty\end{array}$ & $\begin{array}{l}00 \\
00\end{array}$ & $\begin{array}{l}g N \\
\dot{m}\end{array}$ & $\because 0$ & No & $\begin{array}{l}30 \\
\therefore 0\end{array}$ & 눙 & $\begin{array}{l}\text { Lo } \\
\text { fin }\end{array}$ & or & $\ddot{\infty}$ & $\sim m$ & $\forall$ & $\forall \infty$ & [0-7. & -0 & 00 & $m$ \\
\hline 18 & & & & & & & ธิ & {$[0 \infty$} & $\leftrightarrow \infty$ & 닥요 & ind & in: & ్ㅐ, & กิ่ำน & గ్ గ్రి & స్రియ & ஸ̃ & 87 & in \\
\hline 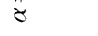 & $\varphi$ & $\begin{array}{l}\infty=1 \\
0-1\end{array}$ & \begin{tabular}{l}
$\operatorname{Ln}$ \\
\hdashline-60
\end{tabular} & $m$ & $m \infty$ & 100 & $\curvearrowright-$ & $\because$ & ช̃. & ION & $\infty$ & $\because$ & N? & $\because-$ & +0 & $\infty 1$ & No. & 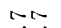 & No \\
\hline & - & $\stackrel{\vec{A}}{ }$ & $6=$ & ర్) & రే) & 冓㐫 & டீ & மூஷ & 대요 & ద2 & 1080 & ถู่ & ถู่อ & ธิ่า & ing & ஜिं & เேं & ज் & ஸิં丶் \\
\hline & 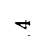 & 00 & $\forall n$ & $\because$ & $\sim \infty$ & $\because 9$ & 100 & 50. & $0 \infty$ & $\rightarrow 0$ & 0 & 09 & $\because$ & ON & $\forall \sim$ & 00 & $\because$ & $\because \because$ & 0. \\
\hline 25 & $-\dot{i}$ & $8 \mathbb{J}$ & 냉을 & $5 \stackrel{9}{=}$ & 冓足 & 奌 & กี่สี่ & in & TE & क्षे & કٓं & $\dot{f}$ & કீं & क⿺ & 舟字 & 宗 & क्षें & ம்ं & मंल \\
\hline & $\vartheta$ & $\begin{array}{l}\sim \infty \\
\sim \infty\end{array}$ & $\begin{array}{l}\infty \sim \\
0\end{array}$ & $10 \infty$ & 207 & O." & No & ON & 09 & N.T. & $\sim-$ & $-\infty$. & $\sigma \pi$ & $\infty \infty$ & $\forall \infty$. & $\because 0$ & $m-$ & $\curvearrowright \dashv$ & 00 \\
\hline & $-i$ & 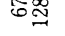 & ช్ูㅗ & $\stackrel{\infty}{\infty} \fallingdotseq$ & 배용 & 표묘 & హి\% & $\mathscr{f}$ & 番题 & 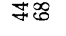 & 80 & $\dot{7} \overrightarrow{0}$ & $\dot{+}+9$ & 舟东 & 요 & ச் & $\dot{\sigma} \dot{\rho}$ & ஒ்ं & ถิล \\
\hline & 0 & $\begin{array}{l}\infty 0 \\
\dot{+}+\dot{+}\end{array}$ & gN & ब. & 70 & -6 & $\theta \vec{*}$ & 0.7 & $\because \tilde{O}$ & $0 .=$ & $\forall$ & $\sim \infty$ & $\sim \sim$ & $m 0$ & $\curlyvee \infty$. & $-\infty$. & gis. & $0 \infty$. & 00 \\
\hline & $-i$ & बल्త్త & గిన్తి & $\vec{W} \vec{\Xi}$ & $\mathscr{1 0}=$ & 98 & ఖి & $\ddot{\forall \infty ~}$ & 98 & मृंతే & $980^{\circ}$ & $\not \dot{q}$ & ゙̛ษ & भुg & 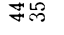 & 守 & q0 & ఖొన్ & హై \\
\hline & $\infty$. & 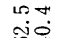 & $\because \overrightarrow{0}$ & $\because$ & 00 & $-\infty$ & $\because$ & ब. & Lo: & 70 & $\forall m$ & $\theta+$ & +0 & OL & 20 & NM & $1 \div$ & 0. & $\sim \sim$ \\
\hline & 0 & Of & ది & LN & $\forall=$ & F。 & ஜீజే & లై & లేవి & மூం & ம் & $\dot{m}$ & 85 & Fल & ن่হ & ホீ心 & భึ & $\Im ה$ & 占 \\
\hline & 0 & $\because \overrightarrow{0}$ & me & $\ddot{O N}$ & $0 N$ & $\rightarrow 0$ & $m \infty$ & $\infty 0$ & $m N$ & $\because$ & $\forall-7$ & $\sim \pi$ & an: & 0. & のम & gis? & - & $\rightarrow N$ & LN \\
\hline & $\circ$ & b & กี & 番兽 & నైల్తు & $m \Xi$ & ని & స̊ & ஸ் & ஜ்ंே் & ్ำช & मెंది & ம் & קైల & ச் & ษ่હ่ & $\dot{y} \infty 0^{\circ}$ & fie & $\dot{f H}$ \\
\hline & & on & to & 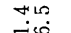 & $\mathfrak{N}$ & $\sim N$ & 00. & $\infty$. N & 00 & ?e & ㅇ. & om & $\infty m$ & $\rightarrow m$ & ? ?- & 09 & बल & 00 & or \\
\hline & $0^{\circ}$ & 经 & ติ & $F \underset{ت}{ }$ & స్లిల్తి & 녹 & నిథ & Iח: & กิน & 刿禺 & నెल & నిః? & गేंत & $\widehat{\infty} \stackrel{\infty}{-}$ & קిల- & ‡भ & ช்่ & ஜேㅇ & $\stackrel{50}{\circ}$ \\
\hline ఈ & $N$ & $\llcorner \%$ & ror. & $\infty$ & 3 & 9. & $0 \infty$ & $\forall \infty$. & mas & ? & $\sim \infty$ & $\because$ & ino. & ๓ & $\sim N$ & m. & $\forall \sim$ & ־ㄴ? & $\because 0$ \\
\hline & $\dot{0}$ & గొ & 平题 & लिప्త్ర & సี & $\approx$ 政 & $=\varnothing$ & $=\infty$ & 禺 & ลีล่ & 20 & శ్లి- & m & ల్లోం & $\infty^{\infty}$ & $F$ & ஜुं & ஜ்ட & Sĭlo \\
\hline & 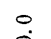 & $\begin{array}{l}0 \\
\text { i̊ }\end{array}$ & $\begin{array}{l}\infty \\
\dot{\phi}+\infty\end{array}$ & i্c) & 노요 & $\vec{m}$ & ஸ் & $\ddot{0}$ & mo & $\stackrel{0}{\circ 0}$ & vंo & So & $\begin{array}{l}\circ \\
\text { mo }\end{array}$ & ن & L & & mo & 100 & 0 \\
\hline$\hat{i}$ & & & & & & & & & & & & d & & & 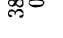 & $7^{\circ}$ & mুo & 20 & $\varphi^{\circ}$ \\
\hline & & $\ddot{0}$ & $\ddot{\circ}$ & $\stackrel{\sim}{0}$ & $\stackrel{\circ}{\circ}$ & $\stackrel{\sim}{\circ}$ & $\ddot{0}$ & $\stackrel{0}{0}$ & $\stackrel{\infty}{0}$ & $\stackrel{\circ}{i}$ & $\stackrel{\sim}{\sim}$ & $\stackrel{\vec{H}}{\sim}$ & $\stackrel{\varphi}{-}$ & $\stackrel{\infty}{-}$ & $\stackrel{\stackrel{\leftrightarrow}{N}}{\mathrm{~N}}$ & $\stackrel{\stackrel{2}{\sim}}{\sim}$ & 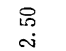 & 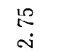 & $\dot{\circ}$ \\
\hline & & 1 & 1 & 1 & & & & & & & & & & & & & & & \\
\hline
\end{tabular}




\begin{tabular}{|c|c|c|c|c|c|c|c|c|c|c|c|c|c|c|c|c|c|c|c|}
\hline \multirow{3}{*}{ 这 } & $\dot{0}$ & मٓं & 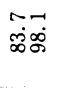 & $\begin{array}{l}\infty+ \\
\dot{\infty} \& \\
\infty\end{array}$ & 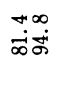 & $\begin{array}{l}\overrightarrow{0} \\
\dot{\infty} \\
\dot{\infty}\end{array}$ & कं & 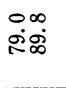 & 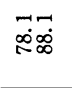 & 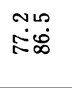 & 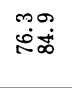 & 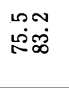 & $\begin{array}{l}00 \\
\dot{+} \dot{0}\end{array}$ & 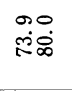 & $\vec{\infty}$ & 氙 & 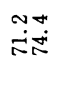 & $\begin{array}{l}\text { +o } \\
\text { 온 }\end{array}$ & :ं० \\
\hline & : & & $\dot{\infty} \infty$ & & & & & & $\Rightarrow$ & $\begin{array}{r}-N \\
\dot{0}\end{array}$ & ம் & $+\infty$ & . & $\begin{array}{l}507 \\
\text { No }\end{array}$ & $\stackrel{\infty}{-\infty}$ & sins & - & कo & \\
\hline & & & めळ & க) & . & চচ & Nक & N & No & $2 \infty$ & $\infty \infty$ & $2 \infty$ & $\sim \infty$ & 2 & Ni & 용 & 8 & 造 & 잉이 \\
\hline & 우 & ஸூ & 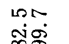 & 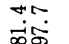 & $\begin{array}{l}\text { Nr. } \\
\dot{\infty} \dot{\S}\end{array}$ & s. & 我 & & $\infty \infty$. & $\begin{aligned} \sim \infty \\
\dot{x}\end{aligned}$ & $\overbrace{\infty \infty}^{\infty}$ & $\infty$ & $\infty \sigma$ & $\vec{\theta}$ & $\overrightarrow{00}$ & - & 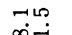 & ma & $\because N$ \\
\hline & & & & & & & & ₹ల & $N \infty$ & $\therefore \infty$ & $2 \infty$ & $\sqrt{\infty}$ & 좃 & 진 & $\approx \pi$ & on & ON & 60 & 86 \\
\hline & $\stackrel{\circ}{+\infty}$ & $\begin{array}{l}\infty \\
\infty \\
\infty\end{array}$ & מn & 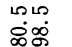 & $\begin{array}{l}\text { Nun } \\
\text { Siष }\end{array}$ & $\begin{array}{l}90 \\
\text { i. } \\
\text { i. }\end{array}$ & - & मूं & $m$ & mit. & స్ & 00 & $0 \infty$ & $\because$ & $\begin{array}{l}\text { No } \\
\infty\end{array}$ & $\begin{array}{l}N-1 \\
\end{array}$ & No & mp & 1509 \\
\hline & & & & & & & & & & & $\therefore \infty$ & & & 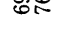 & or & $6 x$ & 88 & ำ & 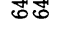 \\
\hline & $\stackrel{\circ}{+}$ & 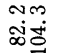 & $\begin{array}{l}\text { ro } \\
\text { ळंள் }\end{array}$ & $\begin{array}{l}\text { mo } \\
\text { siষ্ট }\end{array}$ & 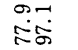 & 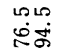 & చN & लें & $\begin{array}{l}\text { LN } \\
\text { Nov }\end{array}$ & 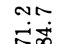 & $\begin{array}{l}0 m \\
0 \infty\end{array}$ & $\begin{array}{l}\infty \% \\
\infty \\
\infty\end{array}$ & 물 & $\begin{array}{l}\text { an } \\
\dot{\phi i n}\end{array}$ & $\begin{array}{l}0 \infty \\
\dot{i n}\end{array}$ & की & $\begin{array}{ll}\text { Om } \\
\dot{x}\end{array}$ & $\vec{r}$ & mm \\
\hline & & $\infty \mathrm{m}$ & $\sim \infty$ & on & $\rightarrow \infty$ & & & & & & & & & & & & & & \\
\hline & $\stackrel{\leftrightarrow}{\infty}$ & க் & ळ் & ஜீ官 & $\dot{\Sigma}$ & ம்ட் & 죠 & 쵸 & $\overrightarrow{i \infty}$ & : & :ं & 80 & \&is & घே: & ड़ंत & ஜ்: & વંષ્ઠ & ธெ & उंड \\
\hline & & NM & 50 & & & & $\rightarrow \infty$ & on & NO & $\infty 0$. & เก & ๑ & $m \infty$. & ๑- & mis & $N+$ & m & 00 & $\infty 0$. \\
\hline & $\dot{m}$ & $\dot{\infty} \dot{0}$ & simg & $\therefore 8$ & 20 & संखी & ฉ்జ & संब्ल & $\dot{\Sigma} \dot{0}$ & $\dot{\infty} \dot{\infty}$ & $\dot{8} \dot{\infty}$ & $\dot{8} \infty$ & ஜேம் & కిંల & rio & ช่: & बंष्ठ & $\dot{80}$ & ำ่ำ \\
\hline & జ & in & $\infty$ & or & $\sim \infty$ & Lor. & ar & mo & no & mo & 요 & $\infty$ & $\cong$ & ro & $\infty \sim$ & $\because$ & $\sigma_{\infty}^{\infty}$ & $\sim \infty$. & 10.7 \\
\hline & $\ddot{m}$ & ఎక్త & 赵苛 & $\approx \vec{\Xi}$ & 20 & నః & స尺 & 요 & 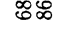 & 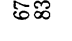 & 80 & Sี & ஜ்ં & ชં่ & ப் & க்க் & ติ่ & मี่ & م⿱⺈ \\
\hline 计h & ì & $\begin{array}{l}\sigma_{\infty} \\
\sigma_{\infty}^{\infty}\end{array}$ & $\varphi_{\infty}^{\infty}$ & or & 70 & $m \sim$ & 100 & $\infty$ & ヘฺฒ & ro. & mo & $\because 0$ & on? & on? & -0 & $\rightarrow N$ & mo. & $\because$ & -0 \\
\hline 祭 & & i. & 표묘 & ㅇ్తి & స৪ & N\& & :ని & i & $5 \infty$ & $\mathscr{\infty}$ & diक & ตㅇ & ત્ષે & घं8 & 800 & मिं & कों & किषें & 호용 \\
\hline 6 & 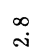 & $\begin{array}{l}\text { No } \\
\text { s. }\end{array}$ & No & $\vec{n}$ & $\overrightarrow{\text { mio. }}$ & $=0$ & $\stackrel{N-1}{*}$ & $+\infty$ & so & -0 & $\infty$ & or & 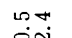 & ம0m. & $\mathscr{O N}$ & $\because 0$ & ON & +0 & $\sigma=1$ \\
\hline 6. & & 묘요 & 욤 & 20. & నిర్తి & స\& & 8ణ & కి̊ & 焽曲 & రేజం & గ్రాన & డొడ & $8 N$ & 88 & ஸீ8 & โกิ & 墭品 & 趈㶽 & 络管 \\
\hline 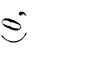 & $\stackrel{\bullet}{i}$ & 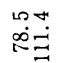 & $\vec{m}=\overrightarrow{0}$ & -0 & 0.0 & $\infty$. & $\infty$ & Oே. & $=\infty$ & Ln? & $\because \infty$ & $\infty$ & $\infty-r$ & $\infty$ & 00. & ヘ & on & $\because 0$. & $\because 0$ \\
\hline$-\nu$ & & & 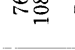 & ボ & ㄷㅇㅛ & 85 & గోః & 乩\% & వుద & $\hat{\sigma} \vec{\infty}$ & $6 \infty$ & ウ゚゙̊ & חה & ins & हिष & 108 & 残战 & 阁敌 & เึ่ \\
\hline$\hat{z}$ & $\stackrel{+}{\sim}$ & 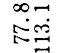 & $\begin{array}{l}\text { mo } \\
\text { ம்: }\end{array}$ & $\begin{array}{l}\infty n \\
\text { Nise }\end{array}$ & 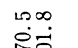 & $\infty \infty$ & -6 & 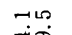 & $m$ & $0 m$ & N? & 0. & or & $\Rightarrow$ & $m \infty$. & $\infty \infty$. & $\because-$ & $\neg \infty$. & $\because n$ \\
\hline$\ddot{3}$ & & & & & No & :55 & $8 \%$ & पైలి & 8 & $\ddot{8 \infty}$ & ํํำ & దొన & 508 & is: & เน็ก & 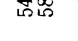 & เైน & คีธี & గొ \\
\hline 6 & $\tilde{N}$ & $\begin{array}{l}90 \\
\dot{8} \pm \\
\dot{y}\end{array}$ & $\begin{array}{l}N= \\
\dot{N}\end{array}$ & تُ. & or & 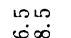 & No & -10 & No & นก, & $\stackrel{\sim}{*}$ & $\infty$ & $g=$ & $\because N$ & Lor & sin & เox & $m$ & $\rightarrow \infty$ \\
\hline <仕 & & & & & & & बळ & & ర్రం & $\infty \infty$ & โ⿵冂卄八 & מתN & 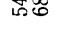 & 苛む゙ & 138 & ్ㅐㅇㅠㅛ & กิกี & 땀여 & 재요 \\
\hline 政 & $\dot{i}$ & 슬 & ن.0 & $\begin{array}{l}0 r \\
\dot{2} \\
\dot{g}\end{array}$ & $\stackrel{N=}{r}$ & Lem? & 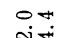 & $\infty$ & $\infty\llcorner 0$ & -6 & ros & $\ddot{0}$ & & $0-7$ & m & 70 & $a-1$ & $\infty 0$. & $\infty 0$. \\
\hline 5 & & & & & & & & & [n & 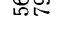 & Wू & ine & ํํㅇ & 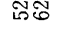 & దำ & เีธ్ర & ํㅛㅇㅛ & 品疋 & గొ \\
\hline$?$ & $\stackrel{\infty}{-}$ & வ்த் & ن를 & 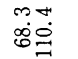 & 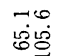 & 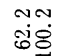 & $\begin{array}{l}n 0 \\
08 \\
0.80\end{array}$ & $\vec{\sim} \dot{\infty}$ & 요 & 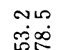 & 오 & $\stackrel{\circ}{+\infty}$ & ma & 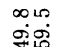 & $\begin{array}{l}1000 \\
810\end{array}$ & $\begin{array}{ll}\sim 7 \\
\dot{9}\end{array}$ & $\begin{array}{lll}N-1 \\
\infty\end{array}$ & mo & サr. \\
\hline${ }_{z}$ & 0 & No & 00 & & & & & & & & & & & & & & & & \\
\hline & - & ฉిస్తి & 8 & เిత & ช่ธ్ڤ & म். & in: & ஸீ & ம் & $\sin$ & 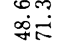 & $\begin{array}{l}\dot{0} 0 \\
\dot{\gamma} \dot{8}\end{array}$ & $\begin{array}{l}+\infty \\
\text { fis }\end{array}$ & 苛密 & $\begin{array}{l}\text { 番 } \\
\dot{y}\end{array}$ & $\begin{array}{l}\text { on } \\
\dot{y}+5\end{array}$ & 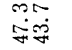 & 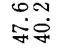 & $\begin{array}{l}\text { om } \\
\text { sis? }\end{array}$ \\
\hline HI & $\nabla$ & go & $\infty N$ & $\infty \infty$ & $m$ & No. & sin. & ก & NN & 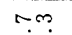 & No & -0 & $\infty 0$. & $\infty \infty$. & 0.0 & mo. & $\infty$ & & $\infty 0$. \\
\hline & - & संష్త & $\dot{\sigma} \dot{\vec{I}}$ & 8용 & ச். & ஸ் & ஸ்่ & ம்க் & 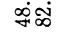 & कें & 字追 & เீ்่ & 东落 & ซึ่ & 字字 & ம்ச & 字宛 & sid & bi \\
\hline & ๆ. & テั & om & $\sigma$. & $m x$ & Nm & $0-1$ & $\infty-$ & $\infty$ & 00 & $\sigma_{\infty}^{\infty}$ & 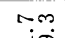 & 必. & กุ? & 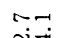 & $\nabla 10$ & $\because$ & $\sigma+$ & or \\
\hline & $-i$ & 욤 & ది & 8.0 & 总气 & 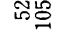 & 㗭。 & \&̊ & অ্ষ & $7 \%$ & F용 & Fin & Fi & $\mathcal{H} \underset{\forall}{\infty}$ & $7 z$ & s;os & హ゙ & హ゙ల్లి & 㘶胥 \\
\hline & & $+\infty$ & $m$ & 90 & mo & $\forall \sim$ & $10 \infty$ & $\varphi$ & $\infty-1$ & 90 & - & -7? & 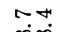 & மon & mo & מেm & $\ddot{\theta}$ & $0 \%$ & L \\
\hline & & జు & రిల్తి & in & 주요 & F。요 & ఖాం & अळ & 冓 & సิ요 & लेढ़ & 串㤁 & 曱文 & 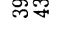 & 강 & $F \vec{~}$ & Fిల & צָล & 我通 \\
\hline & $\stackrel{\infty}{\circ}$ & 여․ & $\therefore N$ & 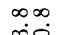 & $\because 7$ & $\infty \varphi$ & $\infty \sigma$ & 100 & 0. & 恶 & 00 & ON & 00 & 0.0 & No? & $\sim \infty$ & -6 & $\forall \sigma$ & $\begin{array}{l}n \infty \infty \\
\text { అூ0 }\end{array}$ \\
\hline & & & $88_{0}^{\circ}$ & గొఝ్తి & ศస్ & $\bar{\nabla} \Xi$ & 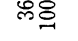 & ాలం & me & స్లిరి & min & 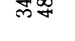 & 岗哥 & 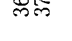 & ణల్లి & గ్రాల్ & デ̊̊ & F్N & జాశ్ \\
\hline & 0 & $\begin{array}{l}+\infty \\
\dot{10} \dot{q}\end{array}$ & ن̃ & & - $\dot{\theta}^{\circ}$ & 100 & $=$ & $?-$ & $5-7$ & $m$ & $\infty$. & -9 & - & $\stackrel{\infty m}{*}$ & $m$ & N" & $a x$ & $\underset{+\infty}{+0}$ & ros \\
\hline & & & & $+\infty$ & & & 周 & సం & NiN & సి & ज्ञ & mf & लె & लైన & ల్లు & চ & 西 & Fこ & 가늘 \\
\hline : & $\stackrel{+}{0}$ & 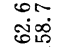 & $\begin{array}{l}+\infty \\
\dot{x}+\underset{+}{+}\end{array}$ & 명 & - & $\vec{\infty}+\infty$ & o.7. & an & 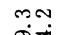 & 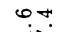 & 0 & Lоm & $0+$ & - & $+\infty$ & $\stackrel{*}{*}$ & 요 & $0 \%$ & $-\infty$ \\
\hline$\tilde{\theta}$ & & & & & - & N & $\overrightarrow{\text { ते }}$ & $-\infty$ & (2) & $\overline{\text { สิ }}$ & స్రాల్ల & సని & దిసే & ల్లైన & 岕二 & $m$ & గ్లిల్త & $s=$ & 가을 \\
\hline & : & $\begin{array}{l}+\infty \\
\dot{\theta} \dot{0} \\
\dot{0}\end{array}$ & $\begin{array}{l}\text { 促 } \\
\text { i் }\end{array}$ & 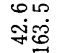 & 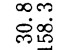 & $\begin{array}{l}6 \\
\dot{*} \\
\dot{*}\end{array}$ & 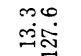 & $\vec{\sigma}_{\infty}^{+}$ & $\begin{array}{l}\ddot{0} 0 \\
\text { ت̇i }\end{array}$ & 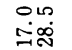 & $\begin{array}{l}+10 \\
\text { تีని }\end{array}$ & تَّف & 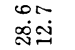 & लि0 & $\begin{array}{l}\infty \\
\text { జ్లై }\end{array}$ & 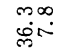 & 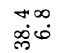 & $\begin{array}{l}-0 \\
\dot{\sigma} \dot{\phi}\end{array}$ & تैं \\
\hline 7 & & & & & & & & 0 & $\because$ & . & 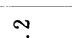 & $\therefore$ & . & 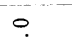 & $\because$ & & $\because$ & ․ & 0. \\
\hline$x$ & 0 & రేఖ & ப் & $\dot{\forall} \approx$ & లివి & $\stackrel{\infty}{\sim}$ & $\infty \underset{\Phi}{\infty}$ & -io & $\infty$ & פृं & నं० & సं० & $\stackrel{\text { \} }{0}} &{\text { लं० }} &{\text { mo }} &{\dot{m} 0} &{\infty_{\infty}^{\circ} 0} &{\text { gio }} &{\text { 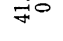 }} \\
{\hline} &{ } &{\begin{array}{l}0 \\
0 \\
1\end{array}} &{\begin{array}{l}\ddot{0} \\
\dot{1}\end{array}} &{\begin{array}{l}\multirow{2}{0}{} \\
0 \\
1\end{array}} &{\ddot{0}} &{\ddot{\sim}} &{\stackrel{+}{0}} &{\stackrel{0}{0}} &{\stackrel{\infty}{0}} &{\stackrel{\circ}{-}} &{\stackrel{\sim}{-}} &{\stackrel{\leftrightarrow}{-}} &{\stackrel{0}{-}} &{\stackrel{\infty}{-}} &{\stackrel{0}{i}} &{\begin{array}{l}\stackrel{\mathscr{N}}{N} \\
\stackrel{N}{N}\end{array}} &{\text { }} &{\text { 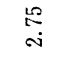 }} &{\stackrel{\circ}{\dot{m}}} \\
$\hline
\end{tabular}

